Article

\title{
A Putative Prophylactic Solution for COVID-19: Development of Novel Multiepitope Vaccine Candidate against SARS-COV-2 by Comprehensive Immunoinformatic and Molecular Modelling Approach
}

\author{
Hafiz Muzzammel Rehman 1,2, Muhammad Usman Mirza ${ }^{3}{ }^{-}$, Mian Azhar Ahmad 2,4, \\ Mahjabeen Saleem ${ }^{1, *}$, Matheus Froeyen ${ }^{3}$, Sarfraz Ahmad ${ }^{5} \mathbb{D}$, Roquyya Gul ${ }^{6}(\mathbb{D}$, \\ Huda Ahmed Alghamdi ${ }^{7}$, Muhammad Shahbaz Aslam ${ }^{1}$, Muhammad Sajjad ${ }^{8}$ (D) and \\ Munir Ahmad Bhinder ${ }^{2}$ D \\ 1 Institute of Biochemistry and Biotechnology, University of the Punjab, Lahore 54590, Punjab, Pakistan; \\ muzzammel.phd.ibb@pu.edu.pk (H.M.R.); shahbaz.ibb@pu.edu.pk (M.S.A.) \\ 2 Department of Human Genetics and Molecular Biology, University of Health Sciences, \\ Lahore 54590, Punjab, Pakistan; drmianazharahmad@gmail.com (M.A.A.); \\ munirbhinder@uhs.edu.pk (M.A.B.) \\ 3 Department of Pharmaceutical and Pharmacological Sciences, Rega Institute for Medical Research, \\ Medicinal Chemistry, University of Leuven, B-3000 Leuven, Belgium; \\ muhammadusman.mirza@kuleuven.be (M.U.M.); mathy.froeyen@kuleuven.be (M.F.) \\ 4 Department of Health, Government of the Punjab, Lahore 54590, Punjab, Pakistan \\ 5 Drug Design and Development Research Group (DDDRG), Department of Chemistry, Faculty of Science, \\ University of Malaya, Kuala Lumpur 50603, Malaysia; sarfraz.ahmad@um.edu.my \\ 6 Faculty of Life Sciences, Gulab Devi Educational Complex, Lahore 54590, Punjab, Pakistan; \\ roquyya.gul@gdec.edu.pk \\ 7 Department of Biology, College of Sciences, King Khalid University, Abha 61413, Saudi Arabia; \\ hudaghamdi@kku.edu.sa \\ 8 School of Biological Sciences, University of the Punjab, Quaid e Azam Campus, \\ Lahore 54590, Punjab, Pakistan; sajjad.sbs@pu.edu.pk \\ * Correspondence: mahjabeen.ibb@pu.edu.pk
}

Received: 17 August 2020; Accepted: 5 September 2020; Published: 18 September 2020

check for updates

Simple Summary: COVID-19, caused by a novel coronavirus, SARS-CoV-2, first emerged in China in December 2019, and then spread around the globe with more than 29 million confirmed infections. Immunoinformatics and molecular modelling techniques are time-efficient methods that are used to accelerate the discovery and design of the candidate peptides for vaccine development against SARS-COV-2. Recently, the use of multiepitope vaccines has proved to be a promising immunization strategy against different viruses and other pathogens. In the current study a comprehensive in silico strategy was used to design stable multiepitope vaccine construct (MVC) from B-cell and T-cell epitopes of essential SARS-CoV-2 proteins which include, spike, main protease, non-structural protein 12 (polymerase), and Nsp13 (helicase) with the help of adjuvants and linkers. Molecular dynamics studies revealed that the MVC displayed favourable molecular interactions with human Toll-like receptors (TLRs), which are known in triggering an innate and adaptive immune response. Furthermore, the MVC was checked for its recombinant production in Escherichia coli using a well-known expression system. The MVC showed a stable three-dimensional structure and could serve as a potential candidate for vaccine production, which warrant further experimental research for validation. 


\begin{abstract}
The outbreak of 2019-novel coronavirus (SARS-CoV-2) that causes severe respiratory infection (COVID-19) has spread in China, and the World Health Organization has declared it a pandemic. However, no approved drug or vaccines are available, and treatment is mainly supportive and through a few repurposed drugs. The urgency of the situation requires the development of SARS-CoV-2-based vaccines. Immunoinformatic and molecular modelling are time-efficient methods that are generally used to accelerate the discovery and design of the candidate peptides for vaccine development. In recent years, the use of multiepitope vaccines has proved to be a promising immunization strategy against viruses and pathogens, thus inducing more comprehensive protective immunity. The current study demonstrated a comprehensive in silico strategy to design stable multiepitope vaccine construct (MVC) from B-cell and T-cell epitopes of essential SARS-CoV-2 proteins with the help of adjuvants and linkers. The integrated molecular dynamics simulations analysis revealed the stability of MVC and its interaction with human Toll-like receptors (TLRs), which trigger an innate and adaptive immune response. Later, the in silico cloning in a known pET28a vector system also estimated the possibility of MVC expression in Escherichia coli. Despite that this study lacks validation of this vaccine construct in terms of its efficacy, the current integrated strategy encompasses the initial multiple epitope vaccine design concepts. After validation, this MVC can be present as a better prophylactic solution against COVID-19.
\end{abstract}

Keywords: COVID-19; SARS-CoV-2; spike protein; multiepitope vaccine; molecular modeling

\title{
1. Introduction
}

The severe acute respiratory syndrome coronavirus 2 (SARS-CoV-2), an enveloped, non-segmented positive-sense RNA virus, causes severe respiratory infection [1]. The ongoing 2019-2020 outbreak of coronavirus disease 2019 (COVID-19) [2-4] has led to 727,435 deaths with 19,687,156 confirmed cases globally as per 10 August 2020, and the World Health Organization (WHO) has declared COVID-19 a global health emergency [5]. Coronaviruses are highly pathogenic viruses and are known to be contagious, which was revealed by the SARS and MERS (Middle East respiratory syndrome) outbreak in 2002 and 2012 [6,7]. The recent SARS-CoV-2 is considered as the seventh known human coronavirus (HCoV) from the same family after 229E, NL63, OC43, HKU1, MERS-CoV, and SARS-CoV [8].

Like other coronaviruses, SARS-CoV-2 is spherical, having a diameter of about $125 \mathrm{~nm}$, and its genome $(\sim 30 \mathrm{~kb})$ contains at least six open reading frames, which encode 16 non-structural proteins and 4 major structural proteins, namely, a spike protein (S), a form of glycoprotein; a membrane protein $(\mathrm{M})$, which consists of the membrane; an envelope protein (E); and a nucleocapsid (N) protein, encoded by the ORFs near the $3^{\prime}$ end of the genome. Among these structural proteins, the spike (S) glycoprotein binds to the cellular receptor angiotensin-converting enzyme 2 (ACE2), and is responsible for causing the viral infection [9]. The S precursor protein of SARS-CoV-2 can be proteolytically cleaved into S1 (685 amino acids) and S2 (588 amino acids) subunits [10]. Owing to the integral role of S protein between viral and host cell membrane interactions, it could be a potential target for developing new SARS-CoV-2 vaccines. Previous studies related to the development of anti-SARS-CoV vaccines and therapeutics that target $S$ protein have already been reported [11-14]. Most of the non-structural proteins play an essential role in viral replication, mainly SARS-CoV-2 main protease (Mpro), also known as chymotrypsin-like protease (3CLpro) [15,16], Nsp13 helicase [17], and the Nsp12 RNA-dependent RNA polymerase [18]. These proteins are also highly conserved among coronaviruses [19].

Owing to the high mortality rate of patients, there is an urgent need to develop vaccines and anti-viral drugs to combat the COVID-19 outbreak. Although phenomenal efforts are in progress in developing vaccines and through repurposing studies [20-22], with the advancement in computational biology, it is now possible to accelerate the drug discovery pipeline and vaccine development [23-25], and these methods have surpassed the conventional methods [26,27]. Although Felipe and coworkers 
also reported a live attenuated vaccine using yellow fever 17D as a vector, that can induce SARS-CoV-2 neutralizing antibodies [28]. Numerous studies have been published related to the B- and T-cell epitope-based vaccine development using in silico immunoinformatics methods [29-36].

Keeping in view the urgency situation of the COVID-19 outbreak, developing an effective vaccine is, therefore, a prime research priority. There are some studies on the development of vaccines against SARS-CoV-2 and some vaccines reached have trial phase as well. It will still require 12 to 18 months to develop an effective vaccine [37]. The current study deals with the modelling of novel multiepitope vaccine for SARS-CoV-2 using a cost-effective integrated immunoinformatics approach. This approach has been proved to be promising against viral diseases caused by viruses like yellow fever [38], SARS-CoV [39], influenza [40], Zika [41], Congo Virus [42], and pathogens including L. donovani [43] and S. pneumoniae [44]. Few multiple epitope vaccine strategies proved to be effective against $H$. pylori infection in the BALB/c mice model [45,46], chronic hepatitis B virus infection [47], and foot-and-mouth disease virus (serotype A) in pigs [48].

In the present study, we effectively designed the multiepitope subunit vaccine construct (MVC) by considering the potential B- and T-cell epitopes of SARS-CoV-2 Spike, Mpro, Nsp-12 polymerase, and Nsp13 helicase proteins. The antigenicity, allergenicity, and physiochemical properties of B- and T-cell epitopes were also measured. Later, the structural analysis of MVC interaction with Toll-like receptors (TLRs) was analyzed through molecular dynamics (MD) simulations and binding free energies were estimated. TLRs establish an important link between innate and adaptive immunity. Engagement of TLR signaling pathways is a promising mechanism for accelerating vaccine responses and is involved in therapeutic immunization against infectious diseases [49]. Thus, the interaction of a multiepitope vaccine construct designed through an integrated modelling approach may trigger innate and specific adaptive immunity by activating TLR signaling pathways and may produce a promising immune response against SARS-CoV-2.

\section{Materials and Methods}

\subsection{Coronavirus Protein Sequences and Structural Information}

The primary amino acid sequences of SARS-CoV-2 main protease (Mpro) (306 amino acids), Nsp12 RNA dependent RNA polymerase (932 amino acids), spike (1237 amino acids), and Nsp13 helicase (601 amino acids) proteins were retrieved from GenBank ID: AHZ13508.1. For structural studies, the crystal structures of recently deposited SARS-CoV-2 Mpro (PDB ID: 6LU7) and spike (PDB ID: 6 VYB) protein were obtained from PDB, while the homology models of SARS-CoV-2 Nsp12 RNA polymerase and Nsp13 helicase were obtained from our recent study [50]. These homology models were generated from templates that showed $99.83 \%$ and $96.08 \%$ identities with SARS-CoV Nsp12 (PDB ID: 6NUR) [18], Nsp13 (PDB ID: 6JYT) [17]. These models showed strikingly similar domain architecture with SARS-CoV and were found to be reliable enough to use in epitope identification studies.

\subsection{Prediction of Linear and Conformational B-Cell Epitopes}

The interaction between the antigenic B-cell epitope and B-lymphocyte causes the B-lymphocytes to differentiate into memory cells and antibody-secreting plasma [51]. B-cell epitope has two significant features, including accessibility to the flexible region and the hydrophilic nature of an immunogen [52]. As per the prediction of Parker hydrophilicity, for surface accessibility, Emini prediction [53], antigenicity scale for Kolaskar and Tongaonkar [54], and flexibility prediction for Karplus and Schulz [55], the analysis was employed arithmetically at IEDB (http://www.iedb.org/). Discontinuous (conformational) epitopes prediction for B-cell was performed using Ellipro from IEDB (http://tools.immuneepitope.org/toolsElliPro/) [56], which used three diverse algorithms such as residues' protrusion index (PI) [57], adjoining clustering residues liable upon PI, and approximation of protein shape [58]. 


\subsection{Prediction of Potential Cytotoxic T-Lymphocyte (CTL) Epitopes}

NetCTL.1.2 server (http://www.cbs.dtu.dk/services/Net CTL) was used to predict the CTL epitopes [59], The eliciting of CTLs happened on the surface of antigen-presenting MHC (major histocompatibility complex) molecules. To assimilate the MHC class I binding, efficiency of TAP transport, and the cleavage of proteasomal C-terminal, NetCTL 1.2 server was employed. HLA (human leukocyte antigen) alleles and peptide lengths both were selected and submitted for prediction of T-cell epitopes as an output. For predicting the TAP transport efficiency, the weight matrix was utilized, while for cleavage of proteasomal C-terminal and MHC class I binding, the ANN (artificial neural network) was employed.

\subsection{Epitope Prediction of Helper T-Cell}

For the prediction of the epitope of helper T-cell, NetMHCII 2.2 Server was used, which gives a 15-mer epitope for human alleles. NetMHCII 2.2 Server uses an artificial neuron network for the prediction of a peptide with human alleles, that is, HLA-DP, HLA-DR, and HLA-DQ [60]. On the basis of receptor interaction, MHC II epitopes were predicted and deduced from IC50 values, as well as the assigned percentile ranks. The peptides that show a strong interaction have an IC50 value of $<50 \mathrm{nM}$, while those having intermediate and low affinity have IC50 values of $<500$ and $<5000$, respectively. Therefore, the percentile rank has a direct relation with IC50 and inverts to the affinity for epitope.

\subsection{Multiepitope Vaccine Designing}

The MVC was designed by connecting the peptide sequences in a successive manner with the help of suitable linkers. The occurrence of overlapping residues amid the B-cell (BCL), HTL, and CTL epitopes was unwavering and epitopes with overlapping regions were used for multiepitope vaccine design. It has been established that human $\beta$-defensins have an important role in presenting the microbial peptides to antigen presenting cells and the inflammatory response, thus enhancing the immunogenicity of the bound antigen; therefore, $\beta$-defensins can be used as adjuvants [61-63]. Recently, mammals' $\beta$-defensin was documented to have a possible role to confer HIV (human immunodeficiency virus) infection as a mucosal adjuvant; consequently, owing to its adjuvant characteristics against viral infection [64], it was chosen and added to the $\mathrm{N}$ - and C-terminal sequences of the vaccine construct. Adjuvants were joined with epitopes at the $\mathrm{N}$ - and C-terminal using the EAAAK linker, whereas intra-CTL epitopes were joined using the AAY linker. After the CTL epitope, HTL epitopes were added next to the CTL epitope using the GPGPG linkers, as used in a previous study [64].

\subsection{Antigenicity and Allergenicity Estimation of the MVC}

To be an effective and safe vaccine candidate, the vaccine candidate should be nonallergic with minimum off-targets effects. The nonallergenic and allergenic behaviors of the MVC were assessed by three servers, AllerTOP V2.0 (http://www.ddg-pharmfac.net/AllerTOP/), AlgPred (http: //www.imtech.res.in/raghava/algpred/) and AllergenFP 1.0 [65]. Out of these, the latter categorizes the protein sequence (input) by a k-nearest neighbor algorithm $(\mathrm{kNN} ; \mathrm{k}=3)$ on the basis of the training set comprising 2210 already known allergens from diverse species and nonallergens $(n=2210)$ from the similar species. The former assimilates the SVM module for the prediction of the allergenic nature of protein with high accuracy. The MAST/MEME allergen motif was examined with the help of MAST (Motif Allignment and Search Tool), and the allergenic nature was allocated if an identical motif was determined.

Evaluation of antigenicity of the MVC was done using two freely available servers, VaxiJen v2.0 (http://www.ddg-pharmfac.net/vaxijen/VaxiJen/VaxiJen.html) [66] and ANTIGENpro (http://scratch. proteomics.ics.uci.edu/), where the latter categorizes the antigen based only on the physio-chemical characteristics of the input protein sequence instead of the sequence placement algorithm. 
The correctness of the server is relatively high and differs between $70 \%$ and $89 \%$ based on the target organism. While the former envisages the entire protein antigenicity based on the results obtained by the protein microarray data analysis, it predicts the antigenicity as being independent of the pathogen, but this approach is sequence-based.

\subsection{Physiochemical Parameters Evaluation}

The vaccine construct sequence was used in ProtParam (http://web.expasy.org/protparam/) [67] to examine its physiochemical properties. The criteria on which the sequences of multiepitope vaccine were examined are theoretical PpI, half-life, instability index, aliphatic index, grand average, and stability profiling of hydropathy.

\subsection{Tertiary Structure Prediction and Refinement of $M V C$}

The final vaccine construct was submitted to structure prediction server known as RaptorX (http://raptorx.uchicago.edu/StructurePrediction/predict/) [68] and I-TASSER [69]. It is an exceptional server for protein 3D structures' predictions on the ab initio method and is able to generate from the template that lies in the twilight zone $(<30 \%)$. It utilizes an exclusive nonlinear context-specific alignment and prospective consistency algorithm. The generated models were evaluated through MolProbity for all-atom contacts and geometry [70]. The model was selected for further refinement through molecular dynamics simulations that showed reliable Ramachandran evaluations.

\subsection{Stability Enhancement of MVC by Disulfide Engineering}

Before moving towards the docking protocol, it is essential to enhance the stability of the model through disulfide engineering. It is a novel concept to introduce disulfide bonds to the modelled protein structure. Consequently, the multiepitope model was employed to the Disulfide by Design 2.0 server [71] to achieve disulfide engineering. The protein model was uploaded to identify the residue pairs, which can be utilized for disulfide engineering. To create the disulfide bonds, four residues were selected to mutate them with cysteine residue by the Disulfide by Design 2.0 server.

\subsection{Molecular Docking of Vaccine Constructs with TLR4}

In order to analyze the binary interaction of MVC with TLRs, protein-protein docking was performed using Cluspro [72,73].The PDB structures of TLR-4 (PDB: 4G8A) and TLR-3 (PDB: 1ZIW) were retrieved from PDB. The multiepitope vaccine model was used as a ligand. Both proteins were prepared accordingly, by removing heteroatoms, and the addition of hydrogens and charges. ClusPro is an automated protein-protein docking server, which generates root mean square deviation (RMSD)-based clustering of 1000 docked conformations. Each representative model is chosen from a cluster of docked models based on the scoring function it uses. The most representative docked conformation from the largest cluster was used for further structural analyses.

\subsection{Molecular Dynamics Simulation for TLRs/MVC Complex}

Molecular dynamics simulations were performed in two steps: (1) a 50 ns MD simulation was performed to optimize and refine 3D model of multiepitope vaccine construct before docking, and (2) a second 50 ns MD simulations to examine the backbone stability of TLRs/MVC complexes. After $\mathrm{MD}$, MD clustering was performed, which typically takes the representative conformation from the largest cluster (within 1 Angstrom deviation). These clusters are generated based on the deviation over the course of total snapshots (in our case, after every 2 ps, which generated a total of 25,000 snapshots). This analysis considers all meaningful conformations over the time of simulation. All simulations were performed by AMBER simulation package 18 using the same protocol as described in previous immunoinformatics studies [30,32]. Briefly, with stepwise minimization and an equilibration procedure, the solvated system in explicit water molecules (TIP3P) was submitted to a production run 
at standard temperature $(300 \mathrm{~K})$ and pressure $(1$ bar). The trajectories were collected after every 2 ps for a complete production run, and the CPPTRAJ module was utilized to analyze trajectories. The MD simulation complexes were analyzed using Chimera 1.14.

\subsection{Codon Adaptation and In Silico Cloning}

The sequence of multiepitope vaccine construct was employed to the online server JCat for reverse translation, and cDNA was obtained, which was submitted for codon optimization [74]. The cDNA was evaluated by codon optimization according to the codon adaptation index (CAI) and GC content of the sequence. The acceptable range of the GC content is $30-70 \%$ and the value of CAI varies from 0 to 1 . The higher value of $\mathrm{CAI}$ indicates a higher level of gene expression [75]. The maximum value of CAI is 1 and is considered ideal, whereas a value of more than 0.8 is also acceptable. After this step, the adapted and optimized sequence of the nucleotides consistent to the design of multiepitope vaccine construct was cloned using the restriction cloning module of SnapGene toll in the vector pET28a (+) of E. coli.

\subsection{In Silico Immune Simulation}

To check the immunogenic potential of the vaccine construct, an in silico immune simulation approach was employed using the C-immsim server [76]. The position specific scoring matrix approach was used by the server for the analysis. The server used three compartments of mammals for immune stimulation, that is, lymph node, thymus, and bone marrow [77]. The defaults constraints for simulation were employed, which are as follows: simulation volume (10), simulation steps (100), random seed (12345), host HLA selection (MHC Class I A0101 allele, B MHC class I B0702, DR MHC class II DRB1_0101 allele), and the time for the injection was set as 1.

\section{Results}

In the present research, plausible T-cell and B-cell epitopes (discontinuous and continuous) from SARS-CoV-2 Spike, Mpro, Nsp12 RNA polymerase (RdRp), and Nsp13 helicase proteins were recognized to design peptide vaccines to counter SARS-CoV-2 infection. Most potential epitopes were selected and joined together with appropriate linkers and adjuvant. The 3D model was generated using various online servers, and a reliable model was used for docking and MD simulation studies. Docking and immuno-informatics method are helpful for the prediction of the binding interaction between TLRs and ligand (multiepitope vaccine) complexes, and analysis was done as these are proven useful tools in identifying novel multiepitope vaccines [23,32].

\subsection{Antigenic B-Cell Epitope Prediction}

Depending on the physicochemical properties of amino acids, which have already been observed in practically determined antigen-based epitopes, Kolaskar and Tongaonkar's approach was used for predicting antigenic epitopes of provided sequences. Seventy-five percent experimental precision has been reported for this approach [54]. Using this method, 11 antigenic peptides with 9-14 amino acid length were observed, including two heptapeptides from SARS-CoV-2 Mpro (Table 1). Likewise, out of 932 amino acids, 37 antigenic peptides were predicted in SARS-CoV-2 Nsp12 polymerase. For RdRp, the length of the antigenic peptides was 6-29 amino acid along, with ten heptapeptides and nine octapeptides (Table 2). For Nsp13 helicase, 18 antigenic peptides were predicted, and the length of the antigenic peptide was 6-38, with 7 hexapeptides (Table 3). For the spike protein, 46 antigenic peptides were predicted from 1273 amino acids (Table 4). 
Table 1. Predicted antigenic B-cell epitopes of severe acute respiratory syndrome coronavirus 2 (SARS-CoV-2) main protease (Mpro).

\begin{tabular}{ccccc}
\hline No. & Start & End & Peptide & Length \\
\hline 1 & 15 & 23 & GCMVQVTCG & 9 \\
2 & 32 & 45 & LDDVVYCPRHVICT & 14 \\
3 & 65 & 72 & NFLVQAGN & 8 \\
4 & 83 & 91 & QNCVLKLKV & 9 \\
5 & 101 & 107 & YKFVRIQ & 7 \\
6 & 111 & 120 & TFSVLACYNG & 10 \\
7 & 123 & 129 & SGVYQCA & 7 \\
8 & 153 & 162 & DYDCVSFCYM & 10 \\
9 & 201 & 212 & TVNVLAWLYAAV & 12 \\
10 & 244 & 253 & QDHVDILGPL & 10 \\
$11^{*}$ & 258 & 271 & GIAVLDMCASLKEL & 14
\end{tabular}

* 11 antigenic sites were predicted from the main protease. The underlined residues were also predicted as cytotoxic T-lymphocyte (CTL) epitope.

Table 2. Predicted antigenic B-cell epitopes of SARS-CoV-2 Nsp12 RNA polymerase.

\begin{tabular}{|c|c|c|c|c|}
\hline No. & Start & End & Peptide & Length \\
\hline 17 & 395 & 400 & CFSVAA & 6 \\
\hline 3 & 50 & 56 & KTNCCRF & 7 \\
\hline 8 & 171 & 177 & ILRVYAN & 7 \\
\hline 10 & 201 & 207 & IVGVLTL & 7 \\
\hline 13 & 327 & 333 & GPLVRKI & 7 \\
\hline 20 & 557 & 563 & VAGVSIC & 7 \\
\hline 21 & 573 & 579 & QKLLKSI & 7 \\
\hline 22 & 585 & 591 & ATVVIGT & 7 \\
\hline 26 & 670 & 676 & GGSLYVK & 7 \\
\hline 28 & 725 & 731 & HRLYECL & 7 \\
\hline 31 & 773 & 779 & QGLVASI & 7 \\
\hline 2 & 28 & 35 & TDVVYRAF & 8 \\
\hline 6 & 125 & 132 & $\overline{\text { ADLVYALR }}$ & 8 \\
\hline 15 & 350 & 357 & ELGVVHNQ & 8 \\
\hline 16 & 369 & 376 & KELLVYAA & 8 \\
\hline 18 & 435 & 442 & VELKHFFF & 8 \\
\hline 23 & 633 & 640 & $\overline{\text { MASLVLAR }}$ & 8 \\
\hline 29 & 744 & 751 & EFYAYLRK & 8 \\
\hline 32 & 783 & 790 & KSVLYYQN & 8 \\
\hline 34 & 825 & 832 & DYVYLPYP & 8 \\
\hline 4 & 67 & 75 & DSYFVVKRH & 9 \\
\hline 25 & 658 & 666 & ECAQVLSEM & 9 \\
\hline 30 & 760 & 768 & DDAVVCFNS & 9 \\
\hline 35 & 839 & 847 & $\overline{\text { GAGCFVDDI }}$ & 9 \\
\hline 36 & 859 & 867 & FVSLAIDAY & 9 \\
\hline 1 & 8 & 17 & LNRVCGVSAA & 10 \\
\hline 27 & 694 & 703 & FNICQAVTAN & 10 \\
\hline 33 & 810 & 819 & HEFCSQHTML & 10 \\
\hline 7 & 144 & 154 & EILVTYNCCDD & 11 \\
\hline 9 & 183 & 193 & RQALLKTVQFC & 11 \\
\hline 14 & 335 & 345 & VDGVPFVVSTG & 11 \\
\hline 24 & 643 & 653 & $\overline{\text { TTCCSLSHRFY }}$ & 11 \\
\hline 5 & 87 & 99 & YNLLKDCPAVAKH & 13 \\
\hline $37 *$ & 878 & 890 & ADVFHLYLQYIRK & 13 \\
\hline 19 & 466 & 482 & IRQLLFVVEVVDKYFDC & 17 \\
\hline 11 & 230 & 248 & $\overline{\text { GVPVVDSYYSLLMPILTLT }}$ & 19 \\
\hline 12 & 295 & 323 & HPNCVNCLDDRCILHCANFNVLFSTVFPP & 29 \\
\hline
\end{tabular}

* 37 antigenic sites were predicted. The underlined residues were also predicted as CTL epitope. 
Table 3. Predicted antigenic B-cell epitopes of SARS-CoV-2 Nsp13 helicase protein.

\begin{tabular}{ccccc}
\hline No. & Start & End & Peptide & Length \\
\hline 3 & 70 & 75 & YYCKSH & 6 \\
5 & 207 & 212 & DAVVYR & 6 \\
10 & 369 & 374 & DIVVFD & 6 \\
11 & 384 & 389 & LSVVNA & 6 \\
13 & 423 & 428 & NSVCRL & 6 \\
15 & 493 & 498 & IGVVRE & 6 \\
17 & 542 & 547 & DYVIFT & 7 \\
12 & 394 & 400 & KHYVYIG & 7 \\
16 & 522 & 528 & ASKILGL & 8 \\
$18 *$ & 570 & 576 & VGILCIM & 9 \\
1 & 4 & 11 & ACVLCNSQ & 9 \\
6 & 222 & 230 & GDYFVLTSH & 10 \\
9 & 353 & 361 & EQYVFCTVN & 10 \\
4 & 78 & 87 & PISFPLCANG & 34 \\
14 & 449 & 458 & VDTVSALVYD & 38 \\
7 & 237 & 250 & APTLVPQEHYVRT & 34 \\
8 & 292 & 325 & AIGLALYYPSARIVYTACSHAAVDALCEKALKYL & \\
2 & 21 & 58 & RRPFLCCKCCYDHVISTSHKLVLSVNPYVCNAPGCDVT & 38 \\
\hline
\end{tabular}

* 18 antigenic sites were predicted. The underlined residues were also predicted as CTL epitope.

Table 4. Predicted antigenic B-cell epitopes of SARS-CoV-2 spike protein.

\begin{tabular}{|c|c|c|c|c|}
\hline No. & Start & End & Peptide & Length \\
\hline 1 & 4 & 18 & FLVLLPLVSSQCVNL & 15 \\
\hline 2 & 34 & 41 & RGVYYPDK & 8 \\
\hline 3 & 44 & 51 & $\overline{\text { RSSVLHST }}$ & 8 \\
\hline 4 & 53 & 60 & $\underline{\text { DLFLPFFS }}$ & 8 \\
\hline 5 & 65 & 70 & $\overline{\text { FHAIHV }}$ & 6 \\
\hline 6 & 81 & 87 & NPVLPFN & 7 \\
\hline 7 & 115 & 121 & QSLLIVN & 7 \\
\hline 8 & 125 & 134 & NVVIKVCEFQ & 10 \\
\hline 9 & 136 & 146 & CNDPFLGVYYH & 11 \\
\hline 10 & 168 & 174 & $\overline{\text { FEYVSQP }}$ & 7 \\
\hline 11 & 210 & 216 & $\overline{I N} L V R D L$ & 7 \\
\hline 12 & 223 & 230 & LEPLVDLP & 8 \\
\hline 13 & 239 & 248 & QTLLALHRSY & 10 \\
\hline 14 & 263 & 270 & AAYYVGYL & 8 \\
\hline 15 & 272 & 278 & $\overline{\text { PRTFLLK }}$ & 7 \\
\hline 16 & 288 & 295 & AVDCALDP & 8 \\
\hline 17 & 333 & 339 & $\overline{\text { TNLCPFG }}$ & 7 \\
\hline 18 & 359 & 371 & SNCVADYSVLYNS & 13 \\
\hline 19 & 376 & 385 & $\overline{\text { TFKCYGVSPT }}$ & 10 \\
\hline 20 & 430 & 435 & TGCVIA & 6 \\
\hline 21 & 488 & 495 & CYFPLQSY & 8 \\
\hline 22 & 505 & 527 & YQPYRVVVLSFELLHAPATVCGP & 23 \\
\hline 23 & 592 & 599 & FGGVSVIT & 8 \\
\hline 24 & 607 & 615 & QVAVLYQDV & 9 \\
\hline 25 & 617 & 627 & $\overline{\text { CTEVPVAIHAD }}$ & 11 \\
\hline 26 & 647 & 653 & AGCLIGA & 7 \\
\hline 27 & 667 & 674 & GAGICASY & 8 \\
\hline 28 & 687 & 693 & VASQSII & 7 \\
\hline 29 & 723 & 730 & $\overline{\text { TTEILPVS }}$ & 8 \\
\hline 30 & 735 & 741 & $\underline{\text { SVDCTMY }}$ & 7 \\
\hline 31 & 750 & 763 & SNLLLQYGSFCTQL & 14 \\
\hline
\end{tabular}


Table 4. Cont.

\begin{tabular}{ccccc}
\hline No. & Start & End & Peptide & Length \\
\hline 32 & 781 & 788 & VFAQVKQI & 8 \\
33 & 803 & 808 & SQILPD & 6 \\
34 & 837 & 843 & YGDCLGD & 7 \\
35 & 847 & 853 & RDLICAQ & 7 \\
36 & 858 & 864 & LTVLPPL & 7 \\
37 & 873 & 880 & YTSALLAG & 8 \\
38 & 959 & 966 & LNTLVKQL & 8 \\
39 & 973 & 979 & ISSVLND & 7 \\
40 & 1003 & 1011 & SLQTYVTQQ & 9 \\
41 & 1030 & 1037 & SECVLGQS & 8 \\
42 & 1057 & 1070 & PHGVVFLHVTYVPA & 14 \\
43 & 1079 & 1085 & PAICHDG & 7 \\
44 & 1123 & 1132 & SGNCDVVIGI & 10 \\
45 & 1174 & 1179 & ASVVNI & 6 \\
$46^{*}$ & 1221 & 1256 & IAGLIAIVMVTIMLCCMTSCCSCLKGCCSCGSCCKF & 36 \\
\hline
\end{tabular}

* 46 antigenic sites were predicted. The underlined residues were also predicted as CTL epitope.

Moreover, Kolaskar and Tongaonkar's approach also projected the highest residual score of each amino acid in all investigated proteins. In SARS-CoV-2 Mpro, 211 out of 306 amino acids have greater than 1.000 residual scores. From position 85 to 91, the antigenic peptide (CVLKLKV) having lysine at the 88th position was identified with a maximal residual score of 1.22. The Nsp12-RdRp has 686 residues out of 932 with a residual score above 1.000, and valine at position 473, in the peptide (LFVVEVV) from 470 to 476 , has a maximum residual score of 1.246. Likewise, for Nsp13 helicase protein, 479 out of 601 amino acids were predicted with a residual score greater than 1.000, and lysine present at the 28th position in an antigenic peptide from 25 to 31 (LCCKCCY) has a maximum residual score of 1.284. For SARS-CoV-2 S protein, 958 out of 1273 amino acids were predicted to have a residual score higher than 1.000, and leucine at position 8 of the antigenic peptide from position 5 to 11 (LVLLPLV) showed a maximal residual score of 1.261.

A graphical depiction of peptides predicted for B cell from investigated SARS-CoV-2 proteins based on sequence position along the $x$-axis and antigenic propensity (AP) as the y-axis is shown in Figures S1-S4. Divergence in AP is related to the length of the sequence. The minimum AP score for Mpro was 0.844 and the maximum AP score was 1.22 (A), while the maximum and minimum AP scores of Nsp12, Nsp13, and S protein were 1.246, 1.284, and 1.261, respectively, and 0.858, 0.893, and 0.866 , respectively.

\subsection{Prediction of Cytotoxic T-Lymphocyte (CTL) Epitopes}

An infected cell having antigen-presentation triggers the T-cell to turn out as an effector cell and kill the infected cells. Cell death or self-destruction is detected after the attack of CTLs on effected cells. The pathogen's peptide fragment and molecule of MHC interact and are exposed on the cell surface of infected cells. CTLs identify the complex of peptide-protein; moreover, as a consequence, infected cells are killed. The processing of fragment of the peptide (antigen), along with its appearance to the T-cell, is achieved through different steps. Peptides are treated in the cytoplasm through proteasome and transferred to the endoplasmic reticulum (ER) later, where MHC is produced. Here, the peptide is transported to the MHC I molecule by the transporter associated with antigen processing (TAP). Afterwards, a complex of peptide-MHC-I is transferred to the surface of the cell. A varied array of peptides is attached to each allelic type of MHC-I protein. The molecule of MHC can interact with peptides strongly as the pathogens attempt to mutate the MHC molecule's epitope. Therefore, the MHC molecule displays strong binding with a diversity of peptides [78]. 
Prediction of CTL epitope is a significant in silico tool in designing the vaccine as it decreases the time and necessity for in vitro trials. NetCTL 1.2 server [59] was used for the prediction of CTL epitope. For all investigated SARS-CoV-2 proteins, the peptide sequences were predicted as CTL epitopes based on three main factors, which include their MHC binding capacity, proteasomal cleavage of the peptide from C-terminal, and affinity for the TAP transporter with the default threshold prediction score being $>0.75000$. Among all the peptides, 11 peptide sequences from $\mathrm{S}$ protein, 4 peptides from Mpro, 19 from Nsp12, and 10 from Nsp13 were selected as CTL epitopes. These CTL epitopes were also predicted as an antigenic site. Hence, these peptides can be considered as potential vaccine candidates (Tables 5-8). A complete list of peptides for these four proteins is also given in Tables S1-S4. 
Table 5. Predicted CTL from SARS-CoV-2 S protein *. TAP, transporter associated with antigen processing.

\begin{tabular}{|c|c|c|c|c|c|c|c|}
\hline $\begin{array}{l}\text { Residue } \\
\text { Number }\end{array}$ & Peptide Sequence & $\begin{array}{l}\text { Predicted MHC } \\
\text { Binding Affinity }\end{array}$ & $\begin{array}{c}\text { Rescale Binding } \\
\text { Affinity }\end{array}$ & $\begin{array}{c}\text { C-Terminal } \\
\text { Cleavage Affinity }\end{array}$ & $\begin{array}{l}\text { TAP Transport } \\
\text { Efficiency }\end{array}$ & Prediction Score & $\begin{array}{l}\text { MHC } \\
\text { Ligand }\end{array}$ \\
\hline 604 & TSNQVAVLY & 0.6559 & 2.7847 & 0.944 & 2.991 & 3.0758 & yes \\
\hline 361 & CVADYSVLY & 0.5348 & 2.2705 & 0.9764 & 3.18 & 2.5759 & yes \\
\hline 733 & KTSVDCTMY & 0.4908 & 2.084 & 0.9649 & 3.016 & 2.3795 & yes \\
\hline 687 & VASQSIIAY & 0.3529 & 1.4986 & 0.9656 & 3.089 & 1.7978136 & yes \\
\hline 136 & CNDPFLGVY & 0.2613 & 1.1095 & 0.69 & 2.45 & 1.3355 & yes \\
\hline 261 & GAAAYYVGY & 0.2253 & 0.9568 & 0.7608 & 2.969 & 1.2194 & yes \\
\hline 357 & RISNCVADY & 0.2106 & 0.8941 & 0.9292 & 3.394 & 1.2032 & yes \\
\hline 285 & ITDAVDCAL & 0.235 & 0.9979 & 0.8708 & 0.79 & 1.168 & yes \\
\hline 1237 & MTSCCSCLK & 0.226 & 0.9595 & 0.7525 & 0.479 & 1.0963 & yes \\
\hline 50 & STQDLFLPF & 0.1974 & 0.8383 & 0.553 & 2.511 & 1.0468 & yes \\
\hline 748 & ECSNLLLQY & 0.1413 & 0.6 & 0.5316 & 2.747 & 0.8171 & yes \\
\hline
\end{tabular}

Table 6. CTL prediction from SARS-CoV-2 Main protease *.

\begin{tabular}{ccccccc}
\hline $\begin{array}{c}\text { Residue } \\
\text { Number }\end{array}$ & Peptide Sequence & $\begin{array}{c}\text { Predicted MHC } \\
\text { Binding Affinity }\end{array}$ & $\begin{array}{c}\text { Rescale Binding } \\
\text { Affinity }\end{array}$ & $\begin{array}{c}\text { C-Terminal } \\
\text { Cleavage Affinity }\end{array}$ & $\begin{array}{c}\text { TAP Transport } \\
\text { Efficiency }\end{array}$ & $\begin{array}{c}\text { MHC } \\
\text { Prediction Score }\end{array}$ \\
\hline 201 & TVNVLAWLY & 0.6255 & 2.6559 & 0.8852 & 2.957 & 2.9365 \\
110 & QTFSVLACY & 0.2625 & 1.1146 & 0.9725 & 2.998 & yes \\
153 & DYDCVSFCY & 0.2097 & 0.8905 & 0.9722 & 0.9722 & 1.4104 \\
93 & TANPKTPKY & 0.1676 & 0.7118 & 0.9755 & 2.1717 & yes \\
\hline
\end{tabular}

* Threshold was set at $>0.75000$. Bold shows the amino acids that were also predicted as antigenic sites.

Table 7. Predicted CTL from SARS-CoV-2 Nsp12 RdRp *

\begin{tabular}{|c|c|c|c|c|c|c|c|}
\hline $\begin{array}{l}\text { Residue } \\
\text { Number }\end{array}$ & Peptide Sequence & $\begin{array}{l}\text { Predicted MHC } \\
\text { Binding Affinity }\end{array}$ & $\begin{array}{c}\text { Rescale Binding } \\
\text { Affinity }\end{array}$ & $\begin{array}{c}\text { C-Terminal } \\
\text { Cleavage Affinity }\end{array}$ & $\begin{array}{l}\text { TAP Transport } \\
\text { Efficiency }\end{array}$ & Prediction Score & $\begin{array}{c}\text { MHC } \\
\text { Ligand }\end{array}$ \\
\hline 738 & DTDFVNEFY & 0.7922 & 3.3634 & 0.8873 & 2.458 & 3.6194 & yes \\
\hline 336 & LSFKELLVY & 0.3898 & 1.6552 & 0.9676 & 3.213 & 1.961 & yes \\
\hline 27 & STDVVYRAF & 0.4019 & 1.7065 & 0.6174 & 2.4 & 1.9191 & yes \\
\hline 859 & FVSLAIDAY & 0.3709 & 1.5746 & 0.7669 & 3.096 & 1.8444 & yes \\
\hline 666 & MVMCGGSLY & 0.3637 & 1.5441 & 0.9482 & 3.008 & 1.8368 & yes \\
\hline
\end{tabular}


Table 7. Cont.

\begin{tabular}{|c|c|c|c|c|c|c|c|}
\hline $\begin{array}{l}\text { Residue } \\
\text { Number }\end{array}$ & Peptide Sequence & $\begin{array}{l}\text { Predicted MHC } \\
\text { Binding Affinity }\end{array}$ & $\begin{array}{l}\text { Rescale Binding } \\
\text { Affinity }\end{array}$ & $\begin{array}{c}\text { C-Terminal } \\
\text { Cleavage Affinity }\end{array}$ & $\begin{array}{l}\text { TAP Transport } \\
\text { Efficiency }\end{array}$ & Prediction Score & $\begin{array}{l}\text { MHC } \\
\text { Ligand }\end{array}$ \\
\hline 758 & LSDDAVVCF & 0.3143 & 1.3345 & 0.9556 & 2.412 & 1.5985 & yes \\
\hline 686 & TTAYANSVF & 0.2963 & 1.258 & 0.4772 & 2.663 & 1.4627 & yes \\
\hline 762 & AVVCFNSTY & 0.2435 & 1.0339 & 0.9754 & 3.146 & 1.3375 & yes \\
\hline 463 & MCDIRQLLF & 0.2518 & 1.0691 & 0.1005 & 2.436 & 1.206 & yes \\
\hline 233 & VVDSYYSLL & 0.2332 & 0.9901 & 0.7134 & 0.834 & 1.1388 & yes \\
\hline 700 & VTANVNALL & 0.2007 & 0.8523 & 0.9705 & 1.166 & 1.0562 & yes \\
\hline 818 & MLVKQGDDY & 0.1793 & 0.7614 & 0.8328 & 3.079 & 1.0403 & yes \\
\hline 823 & GDDYVYLPY & 0.1821 & 0.7733 & 0.8456 & 2.213 & 1.0108 & yes \\
\hline 879 & DVFHLYLQY & 0.1677 & 0.7119 & 0.9529 & 3.013 & 1.0055 & yes \\
\hline 876 & EYADVFHLY & 0.1624 & 0.6894 & 0.9603 & 2.953 & 0.9811 & yes \\
\hline 230 & GVPVVDSYY & 0.1504 & 0.6386 & 0.9521 & 2.923 & 0.9276 & yes \\
\hline 434 & SVELKHFFF & 0.1454 & 0.6176 & 0.9285 & 2.636 & 0.8886 & yes \\
\hline 334 & FVDGVPFVV & 0.1739 & 0.7382 & 0.8437 & 0.191 & 0.8743 & yes \\
\hline 645 & CCSLSHRFY & 0.1586 & 0.6732 & 0.274 & 2.91 & 0.8598 & yes \\
\hline
\end{tabular}

* Threshold was set at $>0.75000$. Bold shows the amino acids that were also predicted as antigenic sites.

Table 8. Predicted CTL from SARS-CoV-2 Nsp13 helicase*.

\begin{tabular}{|c|c|c|c|c|c|c|c|}
\hline $\begin{array}{l}\text { Residue } \\
\text { Number }\end{array}$ & Peptide Sequence & $\begin{array}{l}\text { Predicted MHC } \\
\text { Binding Affinity }\end{array}$ & $\begin{array}{l}\text { Rescale Binding } \\
\text { Affinity }\end{array}$ & $\begin{array}{c}\text { C-Terminal } \\
\text { Cleavage Affinity }\end{array}$ & $\begin{array}{l}\text { TAP Transport } \\
\text { Efficiency }\end{array}$ & Prediction Score & $\begin{array}{c}\text { MHC } \\
\text { Ligand }\end{array}$ \\
\hline 57 & VTDVTQLYL & 0.4708 & 1.9988 & 0.6073 & 0.68 & 2.1239 & yes \\
\hline 56 & DVTDVTQLY & 0.289 & 1.2271 & 0.9651 & 2.704 & 1.5071 & yes \\
\hline 535 & SSQGSEYDY & 0.2761 & 1.1724 & 0.8149 & 2.847 & 1.437 & yes \\
\hline 238 & PTLVPQEHY & 0.1794 & 0.7617 & 0.8719 & 2.595 & 1.0222 & yes \\
\hline 448 & IVDTVSALV & 0.1991 & 0.8453 & 0.8977 & 0.133 & 0.9866 & yes \\
\hline 574 & CIMSDRDLY & 0.1634 & 0.6937 & 0.1836 & 3.125 & 0.8775 & yes \\
\hline 347 & KVNSTLEQY & 0.1391 & 0.5907 & 0.8156 & 2.971 & 0.8616 & yes \\
\hline 245 & HYVRITGLY & 0.1102 & 0.4678 & 0.9598 & 3.009 & 0.7622 & yes \\
\hline 85 & ANGQVFGLY & 0.1141 & 0.4845 & 0.9132 & 2.746 & 0.7588 & yes \\
\hline 538 & GSEYDYVIF & 0.1401 & 0.5947 & 0.3528 & 2.203 & 0.7578 & yes \\
\hline
\end{tabular}




\subsection{Structure-Based Epitope Prediction}

ElliPro was used to predict the epitopes from the 3D structure of proteins [56]. This advanced program is web-based and used to study the correlation among antigenicity, flexibility, and solvent accessibility of proteins' structure. Furthermore, differentiation of predicted epitopes based on interactions of protein-antibody is an essential property of this program. ElliPro measures the PI score (protrusion index), which shows the percent of atoms of proteins that spread beyond the molecular mass/bulk as well as those responsible for binding antibodies. On the basis of the PI score $(>0.7)$, five, three, and two discontinuous epitopes were selected for SARS-CoV-2 S, Nsp13 helicase, and Nsp12 polymerase, respectively, while only one epitope was identified for Mpro that showed PI > 0.7. The graphical illustration of discontinuous epitopes is shown in Figure 1, while number of residues and epitope scores are tabulated in Tables 9-12.
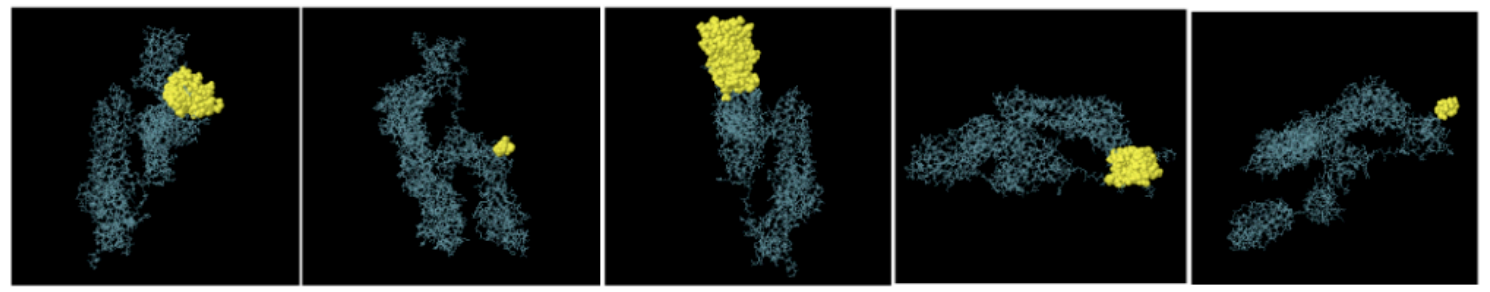

\section{SARS-CoV-2 S protein}

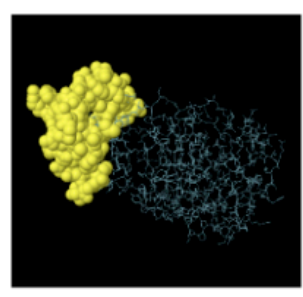

\section{SARS-CoV-2 Mpro}
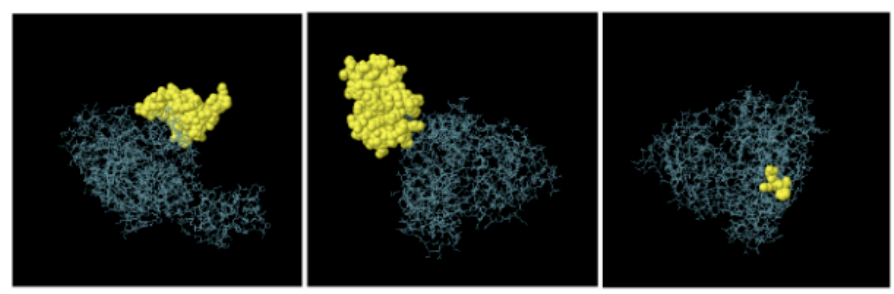

\section{SARS-CoV-2 Nsp12 RNA polymerase}
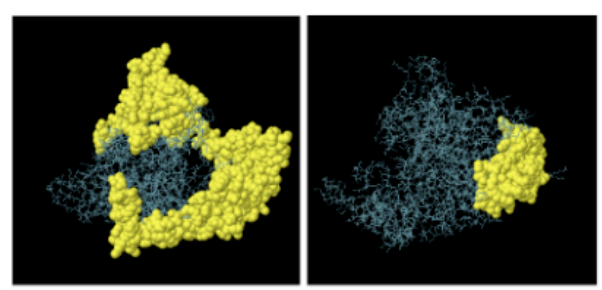

\section{SARS-CoV-2 Nsp13 helicase}

Figure 1. 3D representation of discontinuous epitopes of severe acute respiratory syndrome coronavirus 2 (SARS-CoV-2) spike, Mpro, Nsp12 RNA polymerase, and Nsp13 helicase. 
Table 9. Conformational epitopes of SARS-CoV-2 Mpro.

\begin{tabular}{|c|c|c|c|}
\hline No. & Residues & $\begin{array}{c}\text { Number of } \\
\text { Residues }\end{array}$ & Score \\
\hline 1 & $\begin{array}{l}\text { A:S1, A:G2, A:F3, A:A211, A:V212, A:I213, A:N214, A:G215, A:D216, A:R217, A:W218, A:F219, A:L220, A:N221, A:R222, A:F223, } \\
\text { A:T224, A:T225, A:T226, A:L227, A:N228, A:D229, A:F230, A:N231, A:L232, A:V233, A:A234, A:M235, A:K236, A:Y237, A:Y239, } \\
\text { A:E240, A:P241, A:L242, A:T243, A:Q244, A:D245, A:V247, A:D248, A:L250, A:G251, A:P252, A:S254, A:A255, A:Q256, A:T257, } \\
\text { A:G258, A:I259, A:A260, A:V261, A:L262, A:D263, A:A266, A:S267, A:K269, A:E270, A:L271, A:L272, A:Q273, A:N274, A:G275, } \\
\text { A:M276, A:N277, A:G278, A:R279, A:T280, A:I281, A:L282, A:G283, A:S284, A:A285, A:L286, A:C300, A:S301, A:G302 }\end{array}$ & 75 & 0.716 \\
\hline
\end{tabular}

Table 10. Conformational epitopes of SARS-CoV-2 S protein.

\begin{tabular}{|c|c|c|c|}
\hline No. & Residues & $\begin{array}{l}\text { Number of } \\
\text { Residues }\end{array}$ & Score \\
\hline 1 & $\begin{array}{l}\text { A:L119, A:T120, A:K121, A:Y122, A:T123, A:D126, A:D135, A:E136, A:G137, A:N138, A:C139, A:D140, A:T141, A:K143, A:E144, A:I145, } \\
\text { A:L146, A:V147, A:T148, A:Y149, A:N150, A:C151, A:C152, A:D153, A:D154, A:D155, A:Y156, A:F157, A:N158, A:K159, A:W162, A:Y163, } \\
\text { A:N168, A:P169, A:D170, A:R173, A:V174, A:N177, A:L178, A:E180, A:R181, A:R183, A:Q184, A:A185, A:L187, A:K188, A:T189, A:V190, } \\
\text { A:Q191, A:F192, A:C193, A:D194, A:A195, A:M196, A:R197, A:N198, A:A199, A:G200, A:I201, A:V202, A:G203, A:V204, A:L205, A:T206, } \\
\text { A:D208, A:N209, A:Q210, A:D211, A:L212, A:N213, A:G214, A:N215, A:W216, A:Y217, A:D218, A:F219, A:G220, A:D221, A:F222, A:I223, } \\
\text { A:Q224, A:T225, A:T226, A:P227, A:G228, A:S229, A:G230, A:V231, A:P232, A:V233, A:V234, A:A250, A:D284, A:K288, A:Y289 }\end{array}$ & 95 & 0.728 \\
\hline 2 & 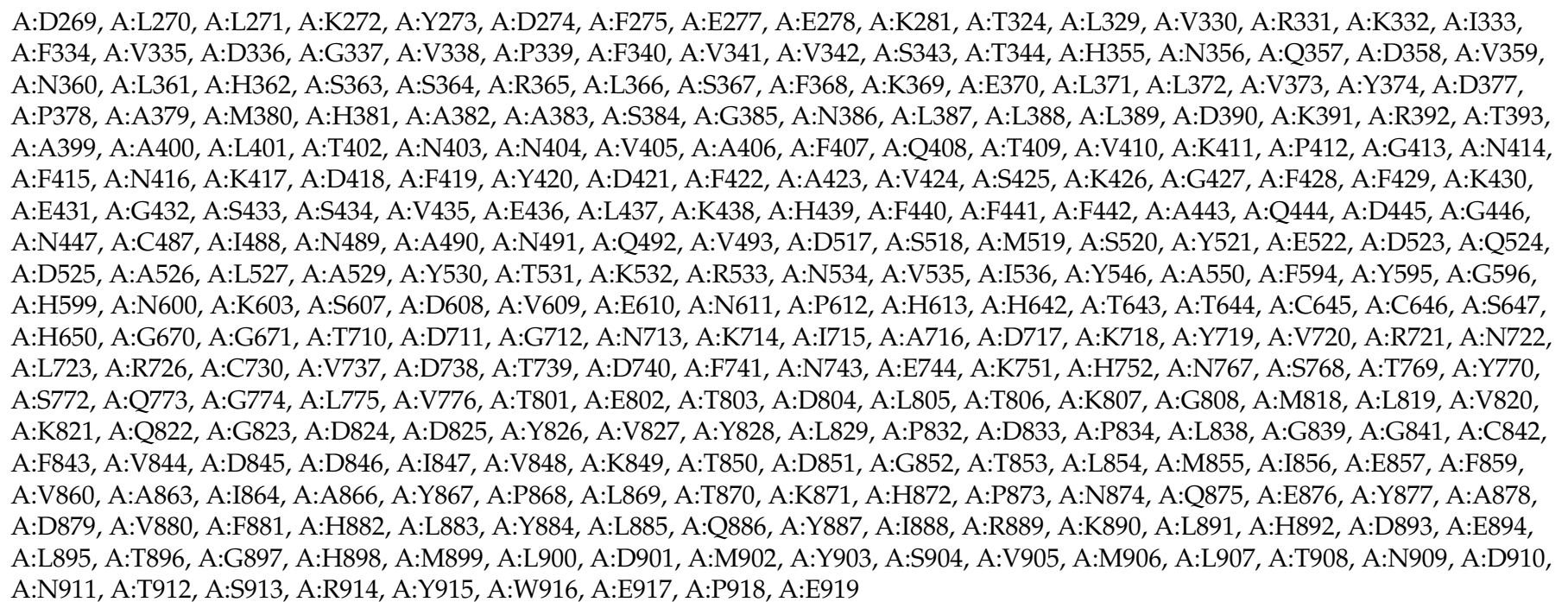 & 297 & 0.719 \\
\hline
\end{tabular}


Table 11. Conformational epitopes from SARS-CoV-2 Nsp12 polymerase.

\begin{tabular}{|c|c|c|c|}
\hline No. & Residues & $\begin{array}{l}\text { Number of } \\
\text { Residues }\end{array}$ & Score \\
\hline 1 & A:D1139, A:P1140, A:L1141, A:Q1142, A:P1143, A:E1144, A:L1145, A:D1146 & 8 & 0.975 \\
\hline 2 & $\begin{array}{l}\text { A:Y707, A:S708, A:N709, A:N710, A:S711, A:I712, A:A713, A:I714, A:P715, A:T716, A:N717, A:Q1071, A:K1073, A:N1074, A:F1075, } \\
\text { A:T1076, A:T1077, A:A1078, A:P1079, A:A1080, A:I1081, A:C1082, A:H1083, A:D1084, A:G1085, A:K1086, A:A1087, A:H1088, A:F1089, } \\
\text { A:P1090, A:R1091, A:E1092, A:G1093, A:V1094, A:F1095, A:V1096, A:S1097, A:N1098, A:G1099, A:T1100, A:H1101, A:W1102, A:F1103, } \\
\text { A:V1104, A:T1105, A:Q1106, A:R1107, A:F1109, A:Y1110, A:E1111, A:P1112, A:Q1113, A:I1114, A:I1115, A:T1116, A:T1117, A:D1118, } \\
\text { A:N1119, A:T1120, A:F1121, A:V1122, A:S1123, A:G1124, A:N1125, A:C1126, A:D1127, A:V1128, A:V1129, A:I1130, A:G1131, A:I1132, } \\
\text { A:V1133, A:N1134, A:N1135, A:T1136, A:V1137, A:Y1138 }\end{array}$ & 77 & 0.845 \\
\hline 3 & $\begin{array}{l}\text { A:L335, A:C336, A:P337, A:F338, A:G339, A:E340, A:V341, A:F342, A:N343, A:A344, A:T345, A:R346, A:F347, A:A348, A:S349, A:V350, } \\
\text { A:Y351, A:A352, A:W353, A:N354, A:R355, A:K356, A:R357, A:I358, A:S359, A:N360, A:C361, A:V362, A:A363, A:D364, A:Y365, A:S366, } \\
\text { A:V367, A:L368, A:Y369, A:N370, A:S371, A:A372, A:S373, A:F374, A:S375, A:T376, A:F377, A:K378, A:C379, A:Y380, A:L390, A:C391, } \\
\text { A:F392, A:T393, A:N394, A:V395, A:Y396, A:A397, A:D398, A:S399, A:F400, A:V401, A:I402, A:R403, A:G404, A:D405, A:E406, A:V407, } \\
\text { A:R408, A:Q409, A:I410, A:A411, A:P412, A:G413, A:Q414, A:T415, A:G416, A:K417, A:I418, A:A419, A:D420, A:Y421, A:N422, A:Y423, } \\
\text { A:K424, A:L425, A:P426, A:D427, A:D428, A:F429, A:T430, A:G431, A:C432, A:V433, A:I434, A:A435, A:W436, A:N437, A:S438, A:N439, } \\
\text { A:N440, A:L441, A:D442, A:S443, A:Y449, A:N450, A:Y451, A:L452, A:Y453, A:R454, A:P491, A:L492, A:Q493, A:S494, A:Y495, A:G496, } \\
\text { A:F497, A:Q498, A:P499, A:T500, A:V503, A:G504, A:Y505, A:Q506, A:P507, A:Y508, A:R509, A:V510, A:V511, A:V512, A:L513, A:S514, } \\
\text { A:F515, A:E516, A:L517, A:L518, A:H519, A:A520, A:P521, A:A522, A:T523, A:V524, A:C525, A:G526, A:P527, A:K528 }\end{array}$ & 142 & 0.799 \\
\hline 4 & A:F559, A:L560, A:P561, A:F562, A:Q563 & 5 & 0.789 \\
\hline 5 & $\begin{array}{l}\text { A:F79, A:D80, A:N81, A:P82, A:V83, A:L84, A:P85, A:I100, A:I101, A:R102, A:G103, A:W104, A:I105, A:T108, A:T109, A:L110, A:D111, } \\
\text { A:S112, A:K113, A:T114, A:Q115, A:S116, A:L117, A:L118, A:I119, A:V120, A:N121, A:N122, A:A123, A:T124, A:N125, A:V126, A:V127, } \\
\text { A:I128, A:K129, A:V130, A:C131, A:E132, A:F133, A:Q134, A:F135, A:C136, A:N137, A:D138, A:P139, A:F140, A:L141, A:G142, A:E156, } \\
\text { A:F157, A:R158, A:V159, A:Y160, A:S161, A:S162, A:A163, A:N164, A:N165, A:C166, A:T167, A:F168, A:E169, A:Y170, A:V171, A:S172, } \\
\text { A:Q173, A:P174, A:F175, A:L176, A:T236, A:R237, A:F238, A:Q239, A:T240, A:L241, A:L242, A:A243, A:L244, A:H245, A:R246 }\end{array}$ & 80 & 0.756 \\
\hline
\end{tabular}


Table 12. Conformational epitopes from SARS-CoV-2 Nsp13 helicase.

\begin{tabular}{|c|c|c|c|}
\hline No. & Residues & $\begin{array}{l}\text { Number of } \\
\text { Residues }\end{array}$ & Score \\
\hline 1 & $\begin{array}{l}\text { A:A1, A:V2, A:G3, A:A4, A:C5, A:L7, A:C8, A:N9, A:S10, A:Q11, A:T12, A:S13, A:L14, A:R15, A:C16, A:G17, A:F24, A:L25, A:C26, A:C27, } \\
\text { A:K28, A:C29, A:C30, A:Y31, A:D32, A:V34, A:I35, A:S36, A:T37, A:S38, A:H39, A:K40, A:L41, A:V42, A:L43, A:S44, A:V45, A:N46, A:P47, } \\
\text { A:Y48, A:V49, A:C50, A:N51, A:A52, A:P53, A:G54, A:C55, A:D56, A:V57, A:T58, A:D59, A:V60, A:T61, A:Q62, A:L63, A:Y64, A:L65, } \\
\text { A:G66, A:G67, A:M68, A:S69, A:Y70, A:Y71, A:C72, A:K73, A:S74, A:H75, A:K76, A:P77, A:P78, A:I79, A:S80, A:F81, A:P82, A:L83, A:C84, } \\
\text { A:A85, A:N86, A:G87, A:Q88, A:V89, A:F90, A:G91, A:L92, A:Y93, A:K94, A:N95, A:T96, A:C97, A:V98, A:G99, A:S100, A:D101, A:N102, } \\
\text { A:V103, A:T104 }\end{array}$ & 96 & 0.761 \\
\hline 2 & A:D344, A:K345, A:F346 & 3 & 0.74 \\
\hline 3 & $\begin{array}{l}\text { A:G150, A:I151, A:A152, A:T153, A:V154, A:R155, A:E156, A:V157, A:L158, A:S159, A:D160, A:R161, A:E162, A:L163, A:H164, A:L165, } \\
\text { A:S166, A:W167, A:E168, A:V169, A:G170, A:K171, A:P172, A:R173, A:G184, A:Y185, A:R186, A:V187, A:T188, A:K189, A:N190, A:S191, } \\
\text { A:K192, A:V193, A:Q194, A:I195, A:G203, A:D204, A:Y205, A:G206, A:D207, A:A208, A:V209, A:Y217, A:K218, A:L219, A:N220, A:V221, } \\
\text { A:G222, A:D223, A:Y224, A:F225 }\end{array}$ & 52 & 0.738 \\
\hline
\end{tabular}




\subsection{Epitope Prediction for (HTL) Helper T Lymphocytes}

MHC class II epitope, which shows high binding affinity, was predicted for human alleles HLA-DP, HLA-DQ, and HLA-DR based on their IC50 values from Net MHC II 2.2 server. These epitopes were described as HTL epitopes. The epitopes with similar sequences were overlapped to get a single epitope. A total of 21 high binding HTL epitopes were selected for the spike (S), main protease, RdRp, and helicase for the novel multiepitope vaccine (Tables S5-S8).

\subsection{Design and Construction of Final Multiepitope Vaccine}

The overlapped and high scoring CTL and HTL epitopes found from SARS-CoV-2 S, Mpro, Nsp12 polymerase, and Nsp13 helicase were combined to form the multiepitope vaccine construct (MVC). To increase the immune response, human $\beta$-defensin 2 (h $\beta D$-2) (PDB ID: 1FD3), the sequence of GIGDPVTCLKSGAICHPVFCPRRYKQIGTCGLPGTKCCKKP and hBD-3 (PDB ID:1KJ6), the sequence of GIINTLQKYYCRVRGGRCAVLSCLPKEEQIGKCSTRGRKCCRRKK were selected as adjuvants at the $\mathrm{N}$ - and C-terminals sequence of the vaccine construct, respectively, with linker EAAK $[79,80]$. After the adjuvant CTL epitopes were combined using appropriate AAY linkers, HTL epitopes were joined together with GPGPG linkers [81] as displayed in Figure S5. By combining potential CTLs, HTLs epitopes, and adjuvants, a multiepitope vaccine construct of 1057 amino acids was constructed.

\subsection{Parametric Evaluation of Physiochemical Properties}

By estimating the multiepitope vaccine construct (MVC) using ProtParam server to estimate physicochemical properties [67], it was found that MVC weighed $114.6 \mathrm{kDa}$. The hypothetical isoelectric point (pI) was 8.15, displaying the basic nature of the MVC, and the assessed in vitro half-life was $30 \mathrm{~h}$ in mammals' reticulocytes [82]. The assessed half-life indicates the time acquired by the protein to remain half of the quantity as originally produced in the cell. The instability index was also predicted to be 35.56 and classified the MVC as stable in nature. The aliphatic index [83] was also examined, which displays the relative volume retained by the aliphatic side chain. It might be reflected as a positive variable for the extension of the thermostability of globular proteins. The attained values of the aliphatic index were found to be 80.93 , indicating that, at varied temperatures, the protein is thermostable. The grand average value of Hydropathy [84] signifies the summation of the hydropathy rate and, along with sequence of amino acid, indicates the hydrophilic and hydrophobic nature of the protein. The observed grand average value of Hydropathy for the vaccine protein was found to be 0.158 .

\subsection{Assessment of Allergenicity and Immunogenicity}

The designed subunit of the vaccine was assessed on the allergenic parameter through AllergenFP 1.0 and AlgPredand AllerTOP 2.0 servers. All these servers predicted the non-allergenic nature of MVC. The antigenicity connected to the vaccine subunit was projected through VaxiJen v2.0 servers. According to the outcome of VaxiJen, the antigenicity of the vaccine was 0.4259 , displaying it as a plausible antigen. Thus, the attained outcome from servers exhibited a high possibility of the subunit vaccine's antigenic and non-allergenic nature.

\subsection{Structure Prediction and Validation of MVC}

In order to analyze the 3D confirmation of MVC, the 1073 amino acid peptide sequence was utilized for the prediction of the 3D model. Multiple softwares were used for modeling, including RaptorX [85] and I-TASSER [86,87], in order to avoid biases. The information of the secondary structure result showed $34 \%$ helical, $19 \% \mathrm{E}$, and $45 \%$ coiled assembly. For homology modeling, the $p$-value is a good parameter to describe the relative quality of the model and a lower $p$-value indicates that the quality is good for the modeled structure. The $p$-value obtained for the MVC structure was $1.29 \times 10^{-6}$, which is lower and significant. For I-TASSER modeling, the model with the highest 
C-score was selected. The models generated from both servers were compared and validated through the MolProbity server [70]. The structure with a better M-score (which combines the clash score, rotamer, and Ramachandran estimations into a single score) was utilized for extensive refinement through MD simulations. The refinement included optimizing bond lengths and angles and removing clashes in geometry [88-90]. The root mean square deviation (RMSD) was calculated for $50 \mathrm{~ns}$. Figure 2A displays the MD refined 3D-model of MVC and the all-backbone RMSD trajectory is shown in Figure 2B. Initially, the RMSD trajectory of MVC model gradually expanded until $30 \mathrm{~ns}$ and reached a value of $\sim 9.5 \AA$. Later, the RMSD value continued to converge until $50 \mathrm{~ns}$, with a deviation $<1 \AA$. This higher RMSD of the simulated model indicated protein expansion during simulation to attain a more stable conformation. The averaged conformation of the MVC model was extracted from the trajectory and compared with the initial model through Ramachandran evaluations. The MD optimized MVC model showed that $86.2 \%$ (924/1073) of all residues were in Ramachandran favoured (>98\%) regions, while the initial homology model showed only $72.49 \%$ (778/1073 residues) in Ramachandran favoured regions. Moreover, residues placed in Ramachandran favoured regions $(>99.8 \%)$ increased from 83.8 (901/1073 residues) with 172 (16.02\%) outliers to 95.5\% (1024/1073) with 49 outliers (4.1\%) (Figure 2C).
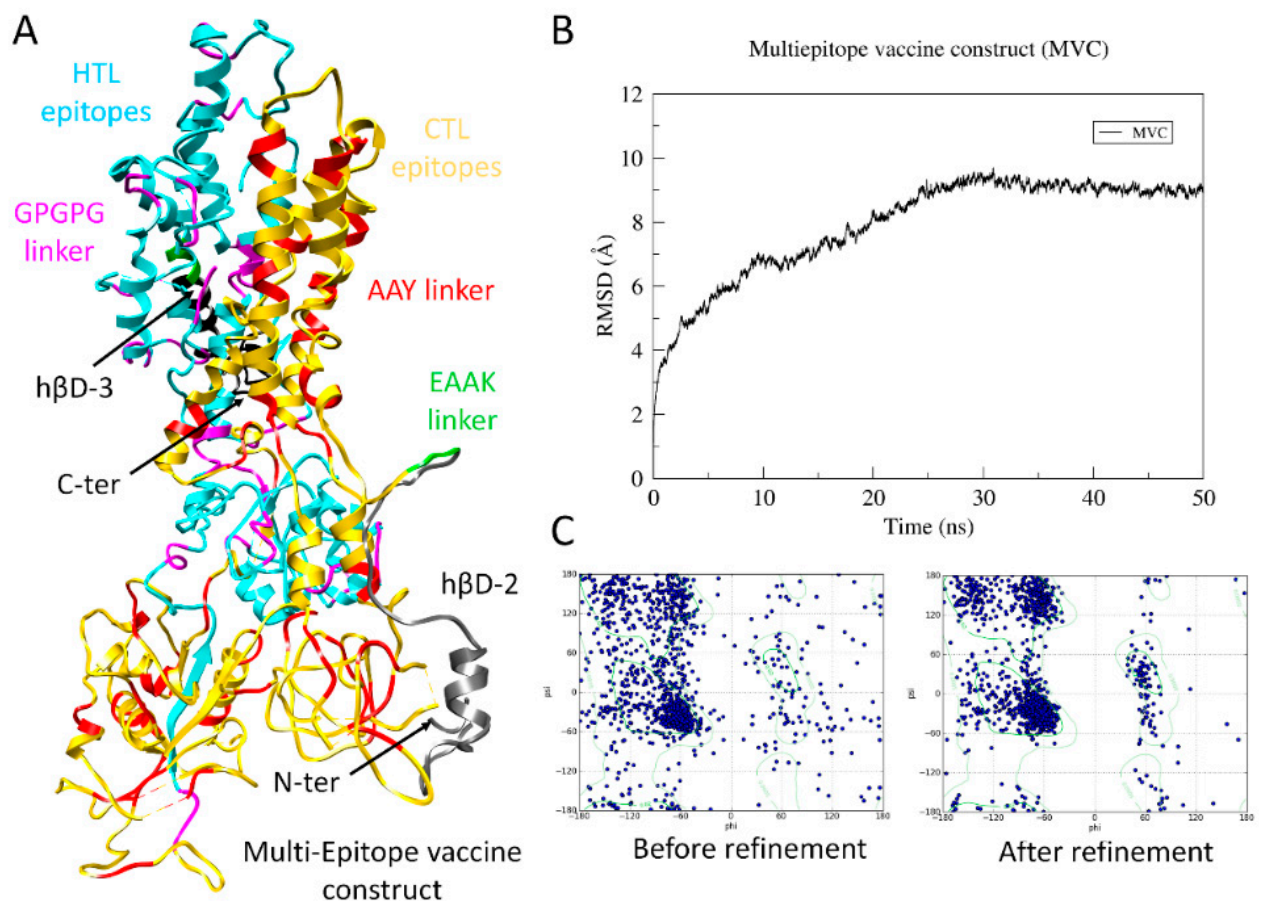

Figure 2. Molecular modeling of vaccine construct. (A) Structural representation of multiepitope vaccine construct (MVC) is displayed with regions (helper T lymphocytes (HTL), cytotoxic T-lymphocyte (CTL) epitopes, linkers, and adjuvants) highlighted accordingly. (B) Root mean square deviation trajectory (RMSD) of MVC analyzed over a period of $50 \mathrm{~ns}$ molecular dynamics (MD) simulations. (C) Ramachandhran evaluations of MVC before and after refinement through MD simulations.

\subsection{Disulfide Engineering for Vaccine Stability}

Disulfide engineering was done to stabilize the modelled structure of MVC, by Disulfide by Design v2.0 server [71]. In the evaluation based on other parameters like Chi3 and energy value, only 07 residues pairs were selected as their value came under the permissible range, that is, energy value must be smaller than 2.2 and Chi3 must be between -87 and +97 degrees. Hence, a total of eight mutations were formed at the pairs of residues, named VAL6-ALA157, TYR138-ALA163, VAL360-GLY730, LEU462-TYR474, ALA499-ARG519, SER814-GLY923, GLY816-SER927, and THR934-GLY946. 


\subsection{Molecular Docking of Vaccine Constructs with TLR3 and TLR4}

Molecular docking is the best in silico approach to finding out interactions between protein-protein and protein-ligand complexes [91-94]. Molecular docking of MVC with TLR4 and TLR3 receptors was performed using ClusPro 2.0, and 30 models were produced [95]. Among these, the complex with the lowest energy was selected. The energy scores attained for TLR3 and TLR4 were -1327.2 and -1270.2, respectively, and subjected to MD simulations to analyze the complex stability. The interaction profile of TLR3 and TLR4 with the MVC showed significant interactions, including H-bonds, salt bridges, and disulfide contacts (Figures S6 and S7). Both hydrogen bonds and salt bridges are particularly important in determining binding specificity [96]. It was observed that MVC established $16 \mathrm{H}$-bonds with TLR3 and 12 H-bonds with TLR4 within the range of 3.00 A (Tables S5-S8).

\subsection{Molecular Dynamics Simulation for TLRS/MVC Complex}

The stability of the TLRs/MVC docked complexes was further investigated by performing MD simulation for a period of $50 \mathrm{~ns}$ in an explicit solvent environment at $300 \mathrm{~K}$. The potential energy of the simulation system was also found to be stable throughout the simulation period (data not provided). The MD refined MVC was utilized for docking, and both complexes showed relatively stable RMSD as compared with MVC alone (Figure 2). In the beginning, the MVC experienced small fluctuations, but remained interacted with the hydrophobic groove of TLR4 (Figure 3A) and TLR3 (Figure 3B), and showed consistent stability in the last $\sim 25 \mathrm{~ns}$. The radius of gyration (RoG) and solvent accessible surface area (SASA) analyses were achieved to determine the compactness $[97,98]$ and protein solvent accessible surface area [99] of TLR3 and TLR4 and designed MVC throughout the MD run (Figure 3C-F). The results suggested similar trends in both complexes. The RoG plot (measured in $\mathrm{nm}$ ) showed no conformational shift, except for small deviations that were evident owing to the flexible linkers utilized, and the overall structure remained stable between 31.5 and $33 \mathrm{~nm}$. The compactness of TLR3 and TLR4 complexed with MVC suggested a strong binding interaction with the designed MVC. A similar description was revealed through SASA analysis (measured in $\mathrm{nm}^{2}$ ), representing the solvent accessible protein surface and its placement through folding, creating the adjustments in the exposed and buried regions of the surface area of proteins. SASA trajectories in both systems also showed a similar trend throughout the simulation period. The presented analysis suggested a stable structure with a significantly strong binding interaction with the vaccine construct, hence providing insights into the biological system's stability [97,98].

\subsection{Codon Adaptation and In Silico Cloning of the MVC}

The reverse translation and codon optimization were performed for the sequence of MVC by the online JCat server [74]. The GC content and codon adaptation index (CAI) were determined out as output from the server. The GC content obtained for MVC was $54.39 \%$, which lies in the acceptable range, that is, from $30 \%$ to $70 \%$. Meanwhile, CAI value of MVC was 1, which indicates a high level of expression in the $\mathrm{K} 12$ strain of E. coli. Later, the restrictions sites of NdeI and XhoI were added and the MVC sequence was cloned in the pET28a (+) vector (Figure 4). The MVC sequence is represented in yellow with the restriction sites. The sequence of multiepitope vaccine construct was cloned between the 6-histidine residue on both sites, which will help in the purification of MVC. 
A

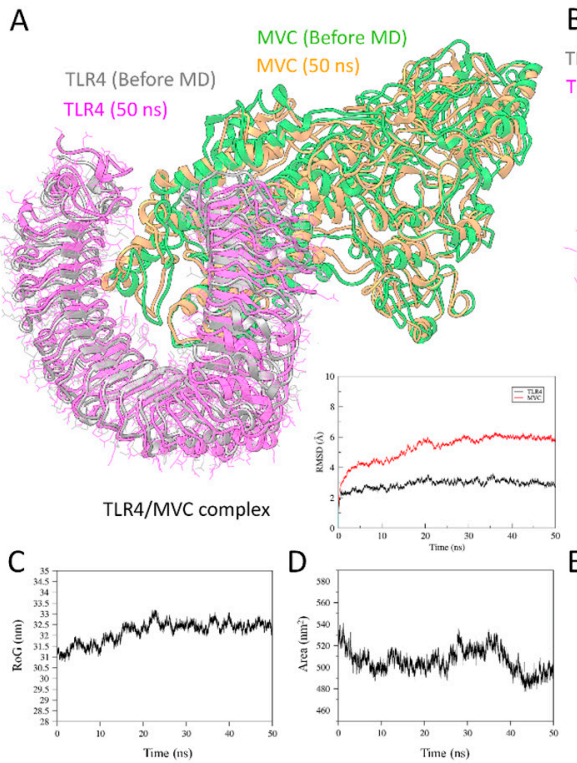

B

LR3 (Before MD) TLR3 (50 ns)
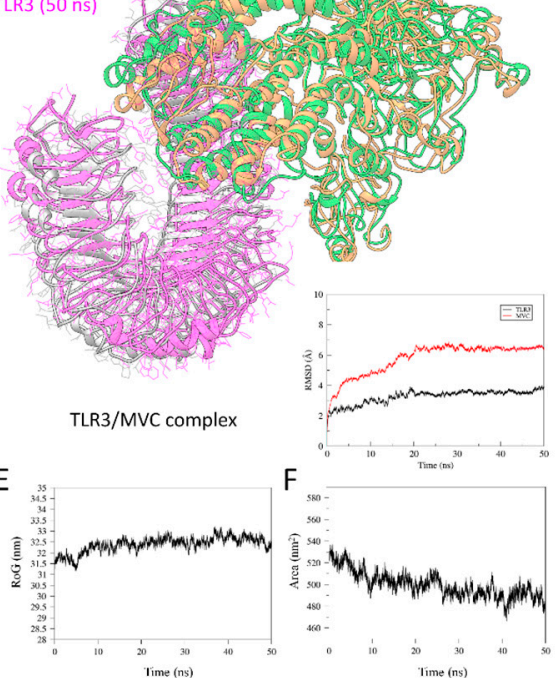

Figure 3. Toll-like receptor (TLR) complexed with a multiepitope vaccine construct (MVC). (A) Conformation of TLR4/MVC and (B) TLR3/MVC complex before and after 50 ns MD simulations, together with the RMSD plot at the bottom indicating the all-atom backbone deviation of TLR (in black) and MVC (in red). (C) Plot of radius of gyration (RoG) and (D) solvent-accessible surface area of TLR4/MVC complex throughout 50 ns MD simulation and TLR3/MVC (E,F).

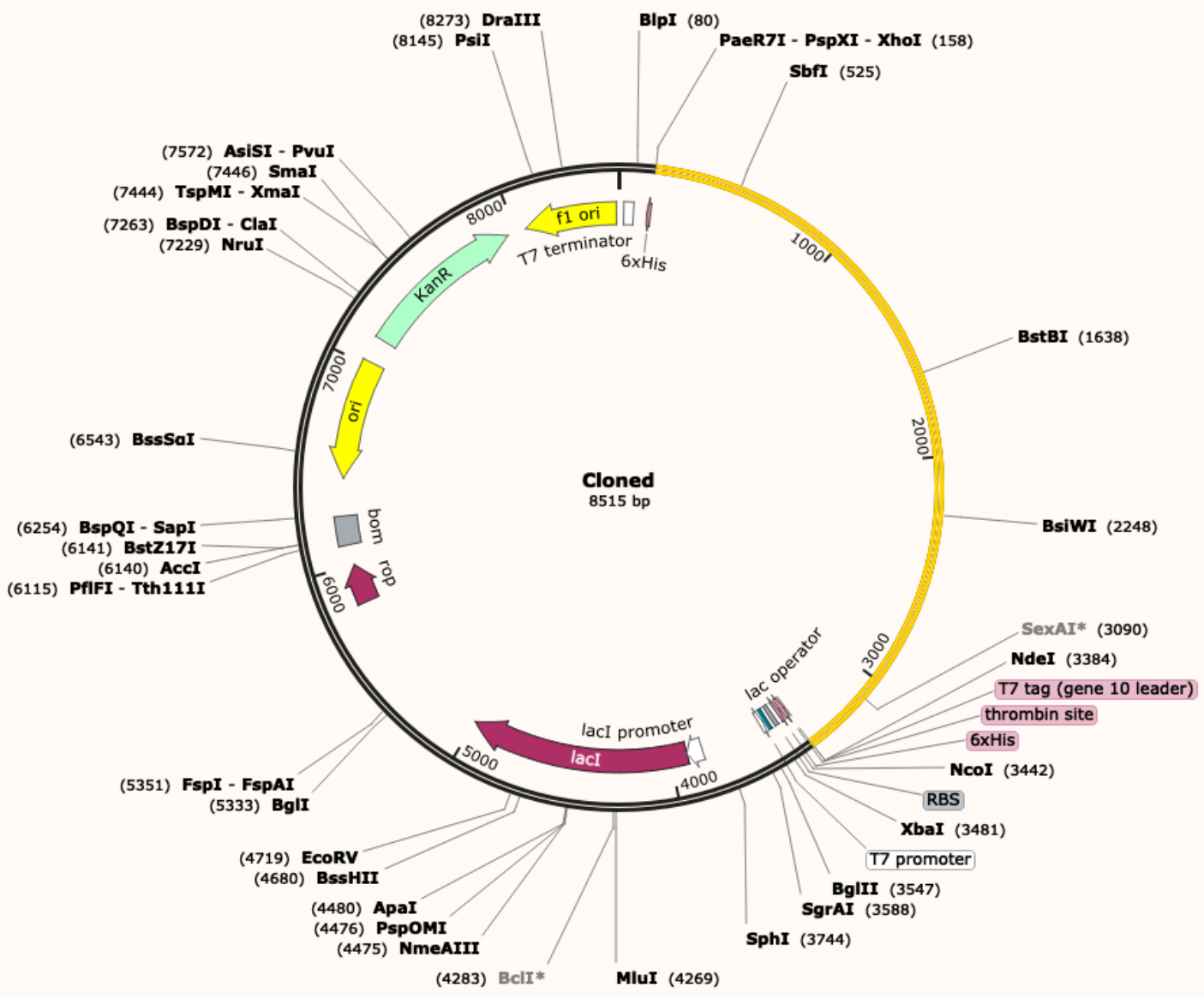

Figure 4. In silico cloning of the multiepitope vaccine construct (MVC). The cDNA of the MVC (yellow) was inserted at the upstream of the T7 promoter. 


\subsection{Immune Simulation by $M V C$}

The immune simulation response of MVC was determined by C-Immsim server. The MVC generated strong primary responses. It has been shown that the titer scale of combined antibodies, IgM and $\mathrm{IgG}$, is approximate to $10,000 / \mathrm{mL}$, and for the antibody, IgM is close to 7000 titer per ml (Figure 5A). The high level of immunoglobulin accomplishments was distinct with associated antigen reduction in both secondary and tertian responses. The level of soluble cytokine, interferon-gamma (IFN-g) was retained, and it was more than $400,000 \mathrm{ng} / \mathrm{mL}$ against the antigen, as shown in Figure 5B.
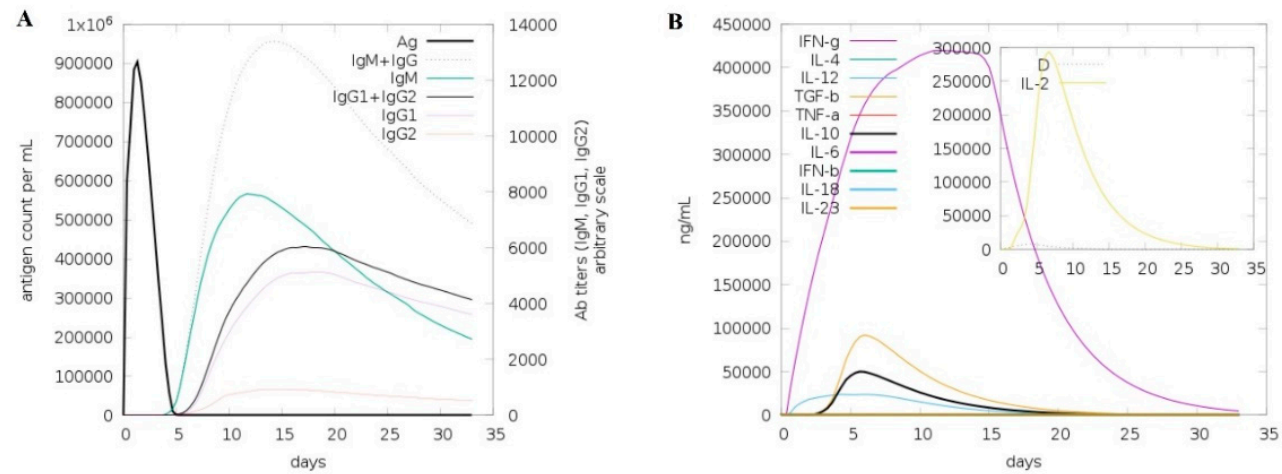

Figure 5. Computational immune simulation by C-Immsim using MVC as antigen. (A) Immunoglobulin/antibodies titer in response to antigen injection. (B) Production of interleukin (IL) and cytokines in response to antigen.

\section{Discussion}

The announcement of emergency by the World Health Organization (WHO) on the COVID-19 outbreak urged researchers to develop therapeutics, mainly the identification of drug candidates or vaccines [20]. The use of cost-effective and less time-consuming methods, especially immunoinformatics approaches, haas already assisted the researchers to predict potential antigenic epitopes for the multiepitope-based vaccine [38-40,44,75,100-102]. The multiple epitope vaccine has a distinctive design concept compared with classical single-epitope based vaccines [101,103-105]. The concept behind the scanning of the viral genome to find immunogenic epitopes leads to an elicited immune response without any reversal of viral pathogenesis [106].

To design a multiepitope vaccine, the research focused on the identification of epitopes for potential $\mathrm{B}$ and $\mathrm{T}$ cells using the immune-informatics approach. An in silico method can be employed using patho-genomics analysis on the genome on a vast scale to identify new vaccines [106,107]. Various limitations are there in the context of appropriate candidate antigens, their immunodominant epitopes, and experimental methods, which include the development of an effective delivery system $[108,109]$. Investigation of the whole spectrum of probable antigens is achievable through immunoinformatics and with the aid of molecular modelling to analyze the potential binding with host proteins $[30,32,38,41,109]$. Besides, the difficulty of culturing the pathogens as well as in vitro antigen expression problems can be avoided [102,110]. Some multiple epitope vaccines showed in vivo efficacy with promising protective immunity $[45,46,48,103]$, while some have entered into phase-I clinical trials, including H2NVAC in patients with HER2-expressing ductal carcinoma in situ (DCIS) (NCT03793829), E1602 for patients with metastatic melanoma [111], EMD640744 in patients with advanced solid tumors [104], and TAB9 in non-HIV-1 infected human volunteers [112]. However, designing an effective multi-epitope vaccine remains a great challenge. Hence, estimation of B cell and CTL cell epitopes by different immune-informatics methods is considered to be a vital tool for designing a multi-epitope construct.

In the present research, potential T-cells and B-cell epitopes (discontinuous and continuous) were recognized from SARS-CoV-2 main protease, Nsp12 RNA polymerase, spike, and Nsp13 helicase proteins to design multi-epitope construct (MVC) using adjuvants (h $\beta$ defensins) and appropriate linkers. The employed linkers (GPGPG and AAY) were carefully selected because their length, 
composition, and structure may affect the activity of the domains and overall characteristics of the molecule [113]. For example, as being somewhat basic antigenic domains (isoelectric point pI > 8), a linker that contains more basic amino acids may increase the pI, such as KK [114]. Therefore, basic linkers were avoided and a glycine-rich linker, that is, GPGPG, was chosen for joining potential epitopes that usually improve solubility and allow the adjoining domains to be accessible and act freely [115]. Following this, a reliable MVC model was generated through molecular modeling and optimized accordingly. All-atom backbone stability of MVC was analyzed through molecular dynamics simulation over a period of $50 \mathrm{~ns}$, because the optimal structural stability of MVC is considered a prime aspect in its efficacy [116], and to the trigger immune response by interacting Toll-like receptors (TLRs) signaling, as successful immunization results are accomplished through stimulation of the TLRs [49]. The resulting model showed fewer outliers, while rotamers were adjusted during the simulation. Molecular docking with TLR3 and TLR4 followed by $50 \mathrm{~ns}$ MD simulation revealed stability in the overall complex in the last $20 \mathrm{~ns}$. The designed MVC interacted with TLR3 and TLR4 directly and their molecular interactions were strengthened during MD simulation, which led to reducing the backbone RMSD fluctuation in both TLR/MVC complexes (Figure 3). However, the epitopes were estimated as non-allergenic, showed antigenicity, and predicted cloning in vector pET28a (+) of E. coli, but given the limitation of in silico tools, the expression and efficacy of the designed multiple vaccine construct should be further proven through in vitro and in vivo experiments.

\section{Conclusions}

COVID-19, after its first emergence in December 2019, widely spread to around 105 countries and the World Health Organization declared it as pandemic. This state of emergency urged to look for effective vaccine candidates and antiviral drugs. The immunoinformatics approach is fast and cost-effective to design and validate the candidate vaccines against such pathogens. In this study, a multiepitope vaccine using spike, Mpro, Nsp-12 polymerase, and Nsp13 helicase proteins of SARS-COV-2 was designed. The epitopes that can induce B- and T-cell mediated immune response were used to build the 3D model of the multiepitope vaccine, which was further validated for its stability and allergenicity. Molecular docking followed by molecular dynamics simulations of MVC with TLR3 and TLR4 was performed, which showed stable interactions of the candidate vaccine with these receptors. Overall, the MVC showed an overall stable structure and could serve as a potential candidate for vaccine production. Although present research is based on an integrated computational approach, further experimental research will be required to validate the effectiveness of the designed vaccine construct.

Supplementary Materials: The following are available online at http://www.mdpi.com/2079-7737/9/9/296/s1, Figure S1: Graphical representation of predicted antigenic propensity of SARS-CoV-2 spike protein; Figure S2: Graphical representation of predicted antigenic propensity of SARS-CoV-2 main protease; Figure S3: Graphical representation of predicted antigenic propensity of SARS-CoV-2 Nsp12 RNA-dependent-RNA-polymerase (RdRp); Figure S4: Graphical representation of predicted antigenic propensity of SARS-CoV-2 Nsp13 helicase; Figure S5: Multiepitope vaccine construct; Figure S6: Molecular interactions of MVC (Chain B) with TLR3 (Chain A); Figure S7: Molecular interactions of MVC (Chain B) with TLR4 (Chain A); Table S1: List of predicted CTL from SARS-CoV-2 S protein; Table S2: List of predicted CTL from SARS-CoV-2 main protease; Table S3: List of predicted CTL from SARS-CoV-2 Nsp12 RdRp; Table S4: List of predicted CTL from SARS-CoV-2 Nsp13 helicase; Table S5: HTL epitope of SARS-CoV-2 main protease (Mpro) protein having strong binding affinity, predicted by NetMHC-II; Table S6: HTL epitope of SARS-CoV-2 RNA polymerase protein having strong binding affinity, predicted by NetMHC-II; Table S7: HTL epitope of SARS-CoV-2 Nsp13 helicase having strong binding affinity, predicted by NetMHC-II; Table S8: HTL epitope of SARS-CoV-2 Spike protein having strong binding affinity, predicted by NetMHC-II; Table S9: List of all molecular interactions of MVC with TLR3; Table S10: List of all molecular interactions of MVC with TLR4.

Author Contributions: H.M.R. and M.U.M. conceived and designed the experiments. H.M.R., M.S. (Muhammad Sajjad), and M.S.A. performed the immunoinformatic and molecular docking analyses. M.U.M. performed the molecular dynamics simulations analysis. M.U.M. and M.A.A. prepared the Figures. M.S. (Mahjabeen Saleem) supervised the study. H.M.R. and M.U.M. wrote the main manuscript text. M.A.A., M.F., S.A., H.A.A., R.G., M.A.B., and M.U.M. critically reviewed the manuscript. All the authors reviewed and approved the final manuscript. All authors have read and agreed to the published version of the manuscript. 
Funding: This research received no external funding.

Acknowledgments: The authors extend their appreciation to the Deanship of Scientific Research at King Khalid University for funding this work through research groups program under Grant No.R.G.P-2/49/40. The authors would like to thank Prof. Dr. Niaz Ahmad Akhtar, Vice chancellor, University of the Punjab, and the Institute of Biochemistry and Biotechnology for providing all necessities in order to complete this work.

Conflicts of Interest: The authors declare no competing interests.

\section{References}

1. Parry, J. China coronavirus: Cases surge as official admits human to human transmission. BMJ 2020, 368, m236. [CrossRef] [PubMed]

2. Benvenuto, D.; Giovanetti, M.; Ciccozzi, A.; Spoto, S.; Angeletti, S.; Ciccozzi, M. The 2019-new Coronavirus epidemic: Evidence for virus evolution. J. Med. Virol. 2020, 92, 455-459. [CrossRef] [PubMed]

3. Wang, C.; Horby, P.W.; Hayden, F.G.; Gao, G.F. A novel coronavirus outbreak of global health concern. Lancet 2020, 395, 470-473. [CrossRef]

4. Perlman, S. Another Decade, Another Coronavirus. 2020, 760-762. [CrossRef]

5. WHO. Coronavirus Disease 2019 (COVID-19) Situation Report-52. Available online: https://www.who. int/docs/default-source/coronaviruse/20200312-sitrep-52-covid-19.pdf?sfvrsn=e2bfc9c0_2 (accessed on 12 March 2020).

6. Drosten, C.; Günther, S.; Preiser, W.; Van Der Werf, S.; Brodt, H.-R.; Becker, S.; Rabenau, H.; Panning, M.; Kolesnikova, L.; Fouchier, R.A.M.; et al. Identification of a novel coronavirus in patients with severe acute respiratory syndrome. N. Engl. J. Med. 2003, 348, 1967-1976. [CrossRef] [PubMed]

7. Azhar, E.I.; Hui, D.S.; Memish, Z.A.; Drosten, C.; Zumla, A. The Middle East Respiratory Syndrome (MERS). Infect. Dis. Clin. 2019, 33, 891-905. [CrossRef] [PubMed]

8. Zhu, N.; Zhang, D.; Wang, W.; Li, X.; Yang, B.; Song, J.; Zhao, X.; Huang, B.; Shi, W.; Lu, R.; et al. A Novel Coronavirus from Patients with Pneumonia in China, 2019. N. Engl. J. Med. 2020, 382, 727-733. [CrossRef]

9. Chen, Y.; Liu, Q.; Guo, D. Emerging Coronaviruses: Genome structure, replication, and pathogenesis. J. Med. Virol. 2020, 92, 418-423. [CrossRef]

10. Chan, J.F.-W.; Kok, K.-H.; Zhu, Z.; Chu, H.; To, K.K.-W.; Yuan, S.; Yuen, K.-Y. Genomic characterization of the 2019 novel human-pathogenic coronavirus isolated from a patient with atypical pneumonia after visiting Wuhan. Emerg. Microbes Infect. 2020, 9, 221-236. [CrossRef]

11. Buchholz, U.J.; Bukreyev, A.; Yang, L.; Lamirande, E.W.; Murphy, B.R.; Subbarao, K.; Collins, P.L. Contributions of the structural proteins of severe acute respiratory syndrome coronavirus to protective immunity. Proc. Nat. Acad. Sci. USA 2004, 101, 9804-9809. [CrossRef]

12. Li, T.; Xie, J.; He, Y.; Fan, H.; Baril, L.; Qiu, Z.; Han, Y.; Xu, W.; Zhang, W.; You, H.; et al. Long-term persistence of robust antibody and cytotoxic $\mathrm{T}$ cell responses in recovered patients infected with SARS coronavirus. PLoS ONE 2006, 1, e24. [CrossRef]

13. Zakhartchouk, A.N.; Sharon, C.; Satkunarajah, M.; Auperin, T.; Viswanathan, S.; Mutwiri, G.; Petric, M.; See, R.H.; Brunham, R.C.; Finlay, B.B.; et al. Immunogenicity of a receptor-binding domain of SARS coronavirus spike protein in mice: Implications for a subunit vaccine. Vaccine 2007, 25, 136-143. [CrossRef] [PubMed]

14. Zhi, Y.; Kobinger, G.P.; Jordan, H.; Suchma, K.; Weiss, S.R.; Shen, H.; Schumer, G.; Gao, G.; Boyer, J.L.; Crystal, R.G.; et al. Identification of murine CD8 T cell epitopes in codon-optimized SARS-associated coronavirus spike protein. Virology 2005, 335, 34-45. [CrossRef] [PubMed]

15. Yang, H.; Xie, W.; Xue, X.; Yang, K.; Ma, J.; Liang, W.; Zhao, Q.; Zhou, Z.; Pei, D.; Ziebuhr, J.; et al. Design of wide-spectrum inhibitors targeting coronavirus main proteases. LoS Biol. 2005, 3.

16. Bacha, U.; Barrila, J.; Velazquez-Campoy, A.; Leavitt, S.A.; Freire, E. Identification of novel inhibitors of the SARS coronavirus main protease 3CLpro. Biochemistry 2004, 43, 4906-4912. [CrossRef] [PubMed]

17. Jia, Z.; Yan, L.; Ren, Z.; Wu, L.; Wang, J.; Guo, J.; Zheng, L.; Ming, Z.; Zhang, L.; Lou, Z.; et al. Delicate structural coordination of the Severe Acute Respiratory Syndrome coronavirus Nsp13 upon ATP hydrolysis. Nucleic Acids Res. 2019, 47, 6538-6550. [CrossRef]

18. Kirchdoerfer, R.N.; Ward, A.B. Structure of the SARS-CoV nsp12 polymerase bound to nsp7 and nsp8 co-factors. Nat. Commun. 2019, 10, 1-9. [CrossRef] [PubMed] 
19. Xu, J.; Zhao, S.; Teng, T.; Abdalla, A.E.; Zhu, W.; Xie, L.; Wang, Y.; Guo, X. Systematic Comparison of Two Animal-to-Human Transmitted Human Coronaviruses: SARS-CoV-2 and SARS-CoV. Viruses 2020, 12, 244. [CrossRef]

20. Morse, J.S.; Lalonde, T.; Xu, S.; Liu, W. Learning from the Past: Possible Urgent Prevention and Treatment Options for Severe Acute Respiratory Infections Caused by 2019-nCoV. Chembiochem 2020, 21, 730-738. [CrossRef]

21. Li, G.; De Clercq, E. Therapeutic options for the 2019 novel coronavirus (2019-nCoV). Nat. Rev. Drug Discov. 2020, 149-150. [CrossRef]

22. Shang, W.; Yang, Y.; Rao, Y.; Rao, X. The outbreak of SARS-CoV-2 pneumonia calls for viral vaccines. NPJ Vaccines 2020, 5, 1-3. [CrossRef] [PubMed]

23. Andaleeb Sajid, S.; Singh, Y.; Shukla, P. Computational tools for modern vaccine development. Hum Vaccin Immunother. 2019.

24. Havranek, B.; Islam, S.M. An in silico approach for identification of novel inhibitors as potential therapeutics targeting COVID-19 main protease. J. Biomol. Struct. Dyn. 2020, 1-12. [CrossRef] [PubMed]

25. Abu-Saleh, A.A.-A.A.; Awad, I.; Yadav, A.; Poirier, R.A. Computational Design of Potent Inhibitors for SARS-CoV-2's Main Protease. ChemRxiv 2020.

26. Arnon, R.; Ben-Yedidia, T. Old and new vaccine approaches. Int. Immunopharmacol. 2003, 3, $1195-1204$. [CrossRef]

27. Hughes, J.P.; Rees, S.; Kalindjian, S.B.; Philpott, K.L. Principles of early drug discovery. Br. J. Pharmacol. 2011, 162, 1239-1249. [CrossRef]

28. Felipe, L.S.; Vercruysse, T.; Sharma, S.; Ma, J.; Lemmens, V.; van Looveren, D.; Javarappa, M.P.A.; Boudewijns, R.; Malengier-Devlies, B.; Kaptein, S.F.; et al. A single-dose live-attenuated YF17D-vectored SARS-CoV2 vaccine candidate. BioRxiv 2020. [CrossRef]

29. Damfo, S.A.; Reche, P.; Gatherer, D.; Flower, D.R. In silico design of knowledge-based Plasmodium falciparum epitope ensemble vaccines. J. Mol. Graph. Model. 2017, 78, 195-205. [CrossRef]

30. Jabbar, B.; Rafique, S.; Salo-Ahen, O.M.; Ali, A.; Munir, M.; Idrees, M.; Mirza, M.U.; Vanmeert, M.; Shah, S.Z.; Jabbar, I. Antigenic Peptide Prediction From E6 and E7 Oncoproteins of HPV Types 16 and 18 for Therapeutic Vaccine Design Using Immunoinformatics and MD Simulation Analysis. Front. Immunol. 2018, 9, 3000. [CrossRef]

31. Michel-Todó, L.; Reche, P.A.; Bigey, P.; Pinazo, M.-J.; Gascón, J.; Alonso-Padilla, J. In silico design of an epitope-based vaccine ensemble for Chagas disease. Front. Immunol. 2019, 10, 2698. [CrossRef]

32. Mirza, M.U.; Rafique, S.; Ali, A.; Munir, M.; Ikram, N.; Manan, A.; Salo-Ahen, O.M.; Idrees, M. Towards peptide vaccines against Zika virus: Immunoinformatics combined with molecular dynamics simulations to predict antigenic epitopes of Zika viral proteins. Sci. Rep. 2016, 6, 37313. [CrossRef] [PubMed]

33. Sabetian, S.; Nezafat, N.; Dorosti, H.; Zarei, M.; Ghasemi, Y. Exploring dengue proteome to design an effective epitope-based vaccine against dengue virus. J. Biomol. Struct. Dyn. 2019, 37, 2546-2563. [CrossRef] [PubMed]

34. Ul Qamar, M.T.; Saleem, S.; Ashfaq, U.A.; Bari, A.; Anwar, F.; Alqahtani, S. Epitope-based peptide vaccine design and target site depiction against Middle East Respiratory Syndrome Coronavirus: An immune-informatics study. J. Transl. Med. 2019, 17, 362. [CrossRef] [PubMed]

35. Kathwate, G.H. In silico design and characterization of multiepitopes vaccine for SARS-CoV2 from its Spike proteins. BioRxiv 2020. [CrossRef]

36. Bhatnager, R.; Bhasin, M.; Arora, J.; Dang, A.S. Epitope based peptide vaccine against SARS-COV2: An immune-informatics approach. J. Biomol. Struct. Dyn. 2020, 1-16. [CrossRef]

37. Green, D.R. SARS-CoV2 Vaccines: Slow is Fast. Am. Assoc. Adv. Sci. 2020. [CrossRef]

38. de Oliveira Tosta, S.F.; Passos, M.S.; Kato, R.; Salgado, Á.; Xavier, J.; Jaiswal, A.K.; Soares, S.C.; Azevedo, V.; Giovanetti, M.; Tiwari, S.; et al. Multi-epitope based vaccine against Yellow fever virus applying immunoinformatics approaches. J. Biomol. Struct. Dyn. 2019, 1-28. [CrossRef]

39. Srivastava, S.; Kamthania, M.; Kumar Pandey, R.; Kumar Saxena, A.; Saxena, V.; Kumar Singh, S.; Kumar Sharma, R.; Sharma, N. Design of novel multi-epitope vaccines against severe acute respiratory syndrome validated through multistage molecular interaction and dynamics. J. Biomol. Struct. Dyn. 2019, 37, 4345-4360. [CrossRef] 
40. Goodman, A.G.; Heinen, P.P.; Guerra, S.; Vijayan, A.; Sorzano, C.O.S.; Gomez, C.E.; Esteban, M. A human multi-epitope recombinant vaccinia virus as a universal $\mathrm{T}$ cell vaccine candidate against influenza virus. PLOS ONE 2011, 6, e25938. [CrossRef]

41. Shahid, F.; Ashfaq, U.A.; Javaid, A.; Khalid, H. Immunoinformatics guided rational design of a next generation multi epitope based peptide (MEBP) vaccine by exploring Zika virus proteome. Infect. Genet. Evol. 2020, 80, 104199. [CrossRef]

42. Nosrati, M.; Behbahani, M.; Mohabatkar, H. Towards the first multi-epitope recombinant vaccine against Crimean-Congo hemorrhagic fever virus: A computer-aided vaccine design approach. J Biomed Inform. 2019, 93, 103160. [CrossRef]

43. Khatoon, N.; Pandey, R.K.; Ojha, R.; Aathmanathan, V.S.; Krishnan, M.; Prajapati, V.K. Exploratory algorithm to devise multi-epitope subunit vaccine by investigating Leishmania donovani membrane proteins. J. Biomol. Struct. Dyn. 2019, 37, 2381-2393. [CrossRef] [PubMed]

44. Dorosti, H.; Eslami, M.; Negahdaripour, M.; Ghoshoon, M.B.; Gholami, A.; Heidari, R.; Dehshahri, A.; Erfani, N.; Nezafat, N.; Ghasemi, Y.; et al. Vaccinomics approach for developing multi-epitope peptide pneumococcal vaccine. J. Biomol. Struct. Dyn. 2019, 37, 3524-3535. [CrossRef] [PubMed]

45. Guo, L.; Yin, R.; Liu, K.; Lv, X.; Li, Y.; Duan, X.; Chu, Y.; Xi, T.; Xing, Y. Immunological features and efficacy of a multi-epitope vaccine CTB-UE against H. pylori in BALB/c mice model. Appl. Microbiol. Biotechnol. 2014, 98, 3495-3507. [CrossRef] [PubMed]

46. Zhou, W.-Y.; Shi, Y.; Wu, C.; Zhang, W.-J.; Mao, X.-H.; Guo, G.; Li, H.-X.; Zou, Q.-M. Therapeutic efficacy of a multi-epitope vaccine against Helicobacter pylori infection in BALB/c mice model. Vaccine 2009, 27, 5013-5019. [CrossRef]

47. Depla, E.; Van der Aa, A.; Livingston, B.D.; Crimi, C.; Allosery, K.; De Brabandere, V.; Krakover, J.; Murthy, S.; Huang, M.; Power, S.; et al. Rational design of a multiepitope vaccine encoding T-lymphocyte epitopes for treatment of chronic hepatitis B virus infections. J. Virol. 2008, 82, 435-450. [CrossRef]

48. Cao, Y.; Li, D.; Fu, Y.; Bai, Q.; Chen, Y.; Bai, X.; Jing, Z.; Sun, P.; Bao, H.; Li, P.; et al. Rational design and efficacy of a multi-epitope recombinant protein vaccine against foot-and-mouth disease virus serotype $\mathrm{A}$ in pigs. Antiviral. Res. 2017, 140, 133-141. [CrossRef]

49. Van Duin, D.; Medzhitov, R.; Shaw, A.C. Triggering TLR signaling in vaccination. Trends Immunol. 2006, 27, 49-55. [CrossRef]

50. Mirza, M.U.; Froeyen, M. Structural Elucidation of SARS-CoV-2 Vital Proteins: Computational Methods Reveal Potential Drug Candidates against Main Protease, Nsp12 RNA-dependent RNA Polymerase and Nsp13 Helicase. J. Pharm. Anal. 2020, 10, 320-328. [CrossRef]

51. Nair, D.T.; Singh, K.; Siddiqui, Z.; Nayak, B.P.; Rao, K.V.; Salunke, D.M. Epitope recognition by diverse antibodies suggests conformational convergence in an antibody response. J. Immunol. 2002, 168, 2371-2382. [CrossRef]

52. Fieser, T.M.; Tainer, J.A.; Geysen, H.M.; Houghten, R.A.; Lerner, R.A. Influence of protein flexibility and peptide conformation on reactivity of monoclonal anti-peptide antibodies with a protein alpha-helix. Proc. Nat. Acad. Sci. USA 1987, 84, 8568-8572. [CrossRef]

53. Emini, E.A.; Hughes, J.V.; Perlow, D.; Boger, J. Induction of hepatitis A virus-neutralizing antibody by a virus-specific synthetic peptide. J. Virol. 1985, 55, 836-839. [CrossRef] [PubMed]

54. Kolaskar, A.; Tongaonkar, P.C. A semi-empirical method for prediction of antigenic determinants on protein antigens. FEBS Lett. 1990, 276, 172-174. [CrossRef]

55. Karplus, P.; Schulz, G. Prediction of chain flexibility in proteins. Naturwissenschaften 1985, 72, $212-213$. [CrossRef]

56. Ponomarenko, J.; Bui, H.-H.; Li, W.; Fusseder, N.; Bourne, P.E.; Sette, A.; Peters, B. ElliPro: A new structure-based tool for the prediction of antibody epitopes. BMC Bioinform. 2008, 9, 514. [CrossRef]

57. Thornton, J.; Edwards, M.; Taylor, W.; Barlow, D. Location of 'continuous' antigenic determinants in the protruding regions of proteins. EMBO J. 1986, 5, 409-413. [CrossRef]

58. Taylor, W.; Thornton, J.t.; Turnell, W. An ellipsoidal approximation of protein shape. J. Mol. Graph. Model. 1983, 1, 30-38. [CrossRef]

59. Larsen, M.V.; Lundegaard, C.; Lamberth, K.; Buus, S.; Lund, O.; Nielsen, M. Large-scale validation of methods for cytotoxic T-lymphocyte epitope prediction. BMC Bioinform. 2007, 8, 424. [CrossRef] 
60. Nielsen, M.; Lund, O. NN-align. An artificial neural network-based alignment algorithm for MHC class II peptide binding prediction. BMC Bioinform. 2009, 10, 296. [CrossRef]

61. Kohlgraf, K.G.; Pingel, L.C.; Dietrich, D.E.; Brogden, K.A. Defensins as anti-inflammatory compounds and mucosal adjuvants. Future Microbiol. 2010, 5, 99-113. [CrossRef]

62. Park, M.S.; Kim, J.I.; Lee, I.; Park, S.; Bae, J.-Y.; Park, M.-S. Towards the application of human defensins as antivirals. Biomol. Ther. (Seoul) 2018, 26, 242. [CrossRef]

63. Weinberg, A.; Jin, G.; Sieg, S.; McCormick, T.S. The yin and yang of human Beta-defensins in health and disease. Front. Immunol. 2012, 3, 294. [CrossRef] [PubMed]

64. Abdulla, F.; Adhikari, U.K.; Uddin, M.K. Exploring T \& B-cell epitopes and designing multi-epitope subunit vaccine targeting integration step of HIV-1 lifecycle using immunoinformatics approach. Microb. Pathog. 2019, 137, 103791. [PubMed]

65. Saha, S.; Raghava, G. AlgPred: Prediction of allergenic proteins and mapping of IgE epitopes. Nucleic Acids Res. 2006, 34, W202-W209. [CrossRef] [PubMed]

66. Doytchinova, I.A.; Flower, D.R. VaxiJen: A server for prediction of protective antigens, tumour antigens and subunit vaccines. BMC Bioinform. 2007, 8, 4. [CrossRef]

67. Gasteiger, E.; Hoogland, C.; Gattiker, A.; Wilkins, M.R.; Appel, R.D.; Bairoch, A. Protein identification and analysis tools on the ExPASy server. In The Proteomics Protocols Handbook; Springer: Berlin/Heidelberg, Germany, 2005; pp. 571-607.

68. Ma, J.; Wang, S.; Zhao, F.; Xu, J. Protein threading using context-specific alignment potential. Bioinformatics 2013, 29, i257-i265. [CrossRef]

69. Zhang, Y. I-TASSER server for protein 3D structure prediction. BMC Bioinform. 2008, 9, 40. [CrossRef]

70. Davis, I.W.; Leaver-Fay, A.; Chen, V.B.; Block, J.N.; Kapral, G.J.; Wang, X.; Murray, L.W.; Arendall III, W.B.; Snoeyink, J.; Richardson, J.S. MolProbity: All-atom contacts and structure validation for proteins and nucleic acids. Nucleic Acids Res. 2007, 35, W375-W383. [CrossRef]

71. Craig, D.B.; Dombkowski, A.A. Disulfide by Design 2.0: A web-based tool for disulfide engineering in proteins. BMC Bioinform. 2013, 14, 346. [CrossRef]

72. Kozakov, D.; Hall, D.R.; Xia, B.; Porter, K.A.; Padhorny, D.; Yueh, C.; Beglov, D.; Vajda, S. The ClusPro web server for protein-protein docking. Nat. Protoc. 2017, 12, 255. [CrossRef]

73. Vajda, S.; Yueh, C.; Beglov, D.; Bohnuud, T.; Mottarella, S.E.; Xia, B.; Hall, D.R.; Kozakov, D. New additions to the C lus P ro server motivated by CAPRI. Proteins 2017, 85, 435-444. [CrossRef]

74. Grote, A.; Hiller, K.; Scheer, M.; Münch, R.; Nörtemann, B.; Hempel, D.C.; Jahn, D. JCat: A novel tool to adapt codon usage of a target gene to its potential expression host. Nucleic Acids Res. 2005, 33, W526-W531. [CrossRef] [PubMed]

75. Nezafat, N.; Eslami, M.; Negahdaripour, M.; Rahbar, M.R.; Ghasemi, Y. Designing an efficient multi-epitope oral vaccine against Helicobacter pylori using immunoinformatics and structural vaccinology approaches. Mol. Syst. Biol. 2017, 13, 699-713. [CrossRef] [PubMed]

76. Rapin, N.; Lund, O.; Castiglione, F. Immune system simulation online. Bioinformatics 2011, 27, $2013-2014$. [CrossRef] [PubMed]

77. Rapin, N.; Lund, O.; Bernaschi, M.; Castiglione, F. Computational immunology meets bioinformatics: The use of prediction tools for molecular binding in the simulation of the immune system. PLoS ONE 2010, 5, e9862. [CrossRef] [PubMed]

78. Alberts, B.; Johnson, A.; Lewis, J.; Raff, M.; Roberts, K.; Walter, P. Molecular Biology of the Cell, 6th ed.; Garland Science: New York, NY, USA, 2002.

79. Nielsen, M.; Lundegaard, C.; Lund, O. Prediction of MHC class II binding affinity using SMM-align, a novel stabilization matrix alignment method. BMC Bioinform. 2007, 8, 238. [CrossRef] [PubMed]

80. Arai, R.; Ueda, H.; Kitayama, A.; Kamiya, N.; Nagamune, T. Design of the linkers which effectively separate domains of a bifunctional fusion protein. Protein Eng. Des. Sel. 2001, 14, 529-532. [CrossRef]

81. Bergmann, C.C.; Yao, Q.; Ho, C.-K.; Buckwold, S.L. Flanking residues alter antigenicity and immunogenicity of multi-unit CTL epitopes. J. Immunol. 1996, 157, 3242-3249.

82. Bachmair, A.; Finley, D.; Varshavsky, A. In vivo half-life of a protein is a function of its amino-terminal residue. Science 1986, 234, 179-186. [CrossRef]

83. Ikai, A. Thermostability and aliphatic index of globular proteins. J. Biochem. 1980, 88, 1895-1898. 
84. Kyte, J.; Doolittle, R.F. A simple method for displaying the hydropathic character of a protein. J. Mol. Biol. 1982, 157, 105-132. [CrossRef]

85. Källberg, M.; Wang, H.; Wang, S.; Peng, J.; Wang, Z.; Lu, H.; Xu, J. Template-based protein structure modeling using the RaptorX web server. Nat. Protoc. 2012, 7, 1511-1522. [CrossRef] [PubMed]

86. Roy, A.; Kucukural, A.; Zhang, Y. I-TASSER: A unified platform for automated protein structure and function prediction. Nat. Protoc. 2010, 5, 725. [CrossRef] [PubMed]

87. Yang, J.; Yan, R.; Roy, A.; Xu, D.; Poisson, J.; Zhang, Y. The I-TASSER Suite: Protein structure and function prediction. Nat. Methods 2015, 12,7-8. [CrossRef] [PubMed]

88. Chen, J.; Brooks, C.L., III. Can molecular dynamics simulations provide high-resolution refinement of protein structure? Proteins 2007, 67, 922-930. [CrossRef] [PubMed]

89. Mirjalili, V.; Noyes, K.; Feig, M. Physics-based protein structure refinement through multiple molecular dynamics trajectories and structure averaging. Proteins 2014, 82, 196-207. [CrossRef]

90. Raval, A.; Piana, S.; Eastwood, M.P.; Dror, R.O.; Shaw, D.E. Refinement of protein structure homology models via long, all-atom molecular dynamics simulations. Proteins 2012, 80, 2071-2079. [CrossRef]

91. Ikram, N.; Mirza, M.U.; Vanmeert, M.; Froeyen, M.; Salo-Ahen, O.M.; Tahir, M.; Qazi, A.; Ahmad, S. Inhibition of Oncogenic Kinases: An In Vitro Validated Computational Approach Identified Potential Multi-Target Anticancer Compounds. Biomolecules 2019, 9, 124. [CrossRef]

92. Iman, K.; Mirza, M.U.; Mazhar, N.; Vanmeert, M.; Irshad, I.; Kamal, M.A. In silico Structure-based Identification of Novel Acetylcholinesterase Inhibitors Against Alzheimer's Disease. CNS Neurol. Disord. Drug Targets 2018, 17, 54-68. [CrossRef]

93. Mirza, M.U.; Ikram, N. Integrated computational approach for virtual hit identification against ebola viral proteins VP35 and VP40. Int. J. Mol. Sci. 2016, 17, 1748. [CrossRef]

94. Mirza, M.U.; Vanmeert, M.; Froeyen, M.; Ali, A.; Rafique, S.; Idrees, M. In silico structural elucidation of RNA-dependent RNA polymerase towards the identification of potential Crimean-Congo Hemorrhagic Fever Virus inhibitors. Sci. Rep. 2019, 9, 1-18. [CrossRef]

95. Comeau, S.R.; Gatchell, D.W.; Vajda, S.; Camacho, C.J. ClusPro: A fully automated algorithm for protein-protein docking. Nucleic Acids Res. 2004, 32, W96-W99. [CrossRef] [PubMed]

96. Xu, D.; Tsai, C.-J.; Nussinov, R. Hydrogen bonds and salt bridges across protein-protein interfaces. Protein Eng. 1997, 10, 999-1012. [CrossRef]

97. McGillewie, L.; Soliman, M.E. The binding landscape of plasmepsin V and the implications for flap dynamics. Mol. Syst. Biol. 2016, 12, 1457-1467.

98. Sindhu, T.; Srinivasan, P. Exploring the binding properties of agonists interacting with human TGR5 using structural modeling, molecular docking and dynamics simulations. RSC Adv. 2015, 5, 14202-14213. [CrossRef]

99. Richmond, T.J. Solvent accessible surface area and excluded volume in proteins: Analytical equations for overlapping spheres and implications for the hydrophobic effect. J. Mol. Biol. 1984, 178, 63-89. [CrossRef]

100. Livingston, B.; Crimi, C.; Newman, M.; Higashimoto, Y.; Appella, E.; Sidney, J.; Sette, A. A rational strategy to design multiepitope immunogens based on multiple Th lymphocyte epitopes. J. Immunol. 2002, 168, 5499-5506. [CrossRef] [PubMed]

101. Saadi, M.; Karkhah, A.; Nouri, H.R. Development of a multi-epitope peptide vaccine inducing robust T cell responses against brucellosis using immunoinformatics based approaches. Infect. Genet. Evol. 2017, 51, 227-234. [CrossRef]

102. Mahmoodi, S.; Nezafat, N.; Barzegar, A.; Negahdaripour, M.; R Nikanfar, A.; Zarghami, N.; Ghasemi, Y. Harnessing bioinformatics for designing a novel multiepitope peptide vaccine against breast cancer. Curr Pharm. Biotechno. 2016, 17, 1100-1114. [CrossRef]

103. Jiang, P.; Cai, Y.; Chen, J.; Ye, X.; Mao, S.; Zhu, S.; Xue, X.; Chen, S.; Zhang, L. Evaluation of tandem Chlamydia trachomatis MOMP multi-epitopes vaccine in BALB/c mice model. Vaccine 2017, 35, 3096-3103. [CrossRef]

104. Lennerz, V.; Gross, S.; Gallerani, E.; Sessa, C.; Mach, N.; Boehm, S.; Hess, D.; Von Boehmer, L.; Knuth, A.; Ochsenbein, A.F.; et al. Immunologic response to the survivin-derived multi-epitope vaccine EMD640744 in patients with advanced solid tumors. Cancer Immunol. Immunother. 2014, 63, 381-394. [CrossRef]

105. Zhu, S.; Feng, Y.; Rao, P.; Xue, X.; Chen, S.; Li, W.; Zhu, G.; Zhang, L. Hepatitis B virus surface antigen as delivery vector can enhance Chlamydia trachomatis MOMP multi-epitope immune response in mice. Appl. Microbiol. Biotechnol. 2014, 98, 4107-4117. [CrossRef] [PubMed] 
106. Zhang, L. Multi-epitope vaccines: A promising strategy against tumors and viral infections. Immunol. Cell Biol. 2018, 15, 182-184. [CrossRef] [PubMed]

107. Rappuoli, R. Reverse vaccinology. Curr. Opin. Microbiol. 2000, 3, 445-450. [CrossRef]

108. Channappanavar, R.; Perlman, S. Evaluation of Activation and Inflammatory Activity of Myeloid Cells during Pathogenic Human Coronavirus Infection. In MERS Coronavirus: Methods and Protocols; Vijay, R., Ed.; Springer US: New York, NY, USA, 2020; pp. 195-204.

109. Yin, D.; Li, L.; Song, X.; Li, H.; Wang, J.; Ju, W.; Qu, X.; Song, D.; Liu, Y.; Meng, X. A novel multi-epitope recombined protein for diagnosis of human brucellosis. BMC Infect. Dis. 2016, 16, 219. [CrossRef]

110. Davies, M.N.; Flower, D.R. Harnessing bioinformatics to discover new vaccines. Drug Discov. Today 2007, 12, 389-395. [CrossRef]

111. Slingluff, C.L.; Lee, S.; Zhao, F.; Chianese-Bullock, K.A.; Olson, W.C.; Butterfield, L.H.; Whiteside, T.L.; Leming, P.D.; Kirkwood, J.M. A randomized phase II trial of multiepitope vaccination with melanoma peptides for cytotoxic T cells and helper T cells for patients with metastatic melanoma (E1602). Clin. Cancer Res. 2013, 19, 4228-4238. [CrossRef]

112. Toledo, H.; Baly, A.; Castro, O.; Resik, S.; Laferté, J.; Rolo, F.; Navea, L.; Lobaina, L.; Cruz, O.; Míguez, J.; et al. A phase I clinical trial of a multi-epitope polypeptide TAB9 combined with Montanide ISA 720 adjuvant in non-HIV-1 infected human volunteers. Vaccine 2001, 19, 4328-4336. [CrossRef]

113. Yu, K.; Liu, C.; Kim, B.-G.; Lee, D.-Y. Synthetic fusion protein design and applications. Biotechnol. Adv. 2015, 33, 155-164. [CrossRef]

114. Yano, A.; Onozuka, A.; Asahi-Ozaki, Y.; Imai, S.; Hanada, N.; Miwa, Y.; Nisizawa, T. An ingenious design for peptide vaccines. Vaccine 2005, 23, 2322-2326. [CrossRef]

115. Kavoosi, M.; Creagh, A.L.; Kilburn, D.G.; Haynes, C.A. Strategy for selecting and characterizing linker peptides for CBM9-tagged fusion proteins expressed in Escherichia coli. Biotechnol. Bioeng. 2007, 98, 599-610. [CrossRef]

116. Scheiblhofer, S.; Laimer, J.; Machado, Y.; Weiss, R.; Thalhamer, J. Influence of protein fold stability on immunogenicity and its implications for vaccine design. Expert Rev. Vaccines 2017, 16, 479-489. [CrossRef] [PubMed] 